\title{
Dualities and inhomogeneous phases in dense quark matter with chiral and isospin imbalances in the framework of effective model
}

\author{
T.G. Khunjua, ${ }^{a}$ K.G. Klimenko ${ }^{b}$ and R.N. Zhokhov ${ }^{c}$ \\ ${ }^{a}$ Faculty of Physics, Moscow State University, \\ 119991, Moscow, Russia \\ ${ }^{b}$ Logunov Institute for High Energy Physics, NRC "Kurchatov Institute", \\ 142281, Protvino, Moscow Region, Russia \\ ${ }^{c}$ Pushkov Institute of Terrestrial Magnetism, Ionosphere and Radiowave Propagation (IZMIRAN), \\ 108840 Troitsk, Moscow, Russia
}

E-mail: gtamaz@gmail.com, kklim@ihep.ru, zhokhovr@gmail.com

ABSTRACT: It has been shown in $[15,70]$ in the framework of Nambu-Jona-Lasinio model with the assumption of spatially homogeneous condensates that in the large- $N_{c}$ limit $\left(N_{c}\right.$ is the number of quark colours) there exist three dual symmetries of the thermodynamic potential, which describes dense quark matter with chiral and isospin imbalances. The main duality is between the chiral symmetry breaking and the charged pion condensation phenomena. There have been a lot of studies and hints that the ground state could be characterized by spatially inhomogeneous condensates, so the question arises if duality is a rather deep property of the phase structure or just accidental property in the homogeneous case. In this paper we have shown that even if the phase diagram contains phases with spatially inhomogeneous condensates, it still possesses the property of this main duality. Two other dual symmetries are not realized in the theory if it is investigated within an inhomogeneous approach to a ground state. Based on various previously studied aspects of the QCD phase diagram of dense isospin asymmetric matter with possible inhomogeneous condensates, in the present paper a unified picture and full phase diagram of dense quark matter with isospin imbalance have been assembled. Acting on this diagram by a dual transformation, we obtained, in the framework of an approach with spatially inhomogeneous condensates and without any calculations, a full phase diagram of chirally asymmetric dense medium. This example shows that the duality is not just entertaining mathematical property but an instrument with very high predictivity power. The obtained phase diagram is quite rich and contains various spatially inhomogeneous phases.

Keywords: Phase Diagram of QCD, Chiral Lagrangians

ARXIV EPRINT: 1901.02855 


\section{Contents}

1 Introduction 1

2 The model and thermodynamic potential $\quad 6$

3 Duality between CSB and CPC phenomena 10

$\begin{array}{lll}3.1 & \text { Spatially homogeneous approach to condensates } & 10\end{array}$

$\begin{array}{ll}3.2 & \text { Duality in inhomogeneous case } \\ 3.3 & \text { Duality and }\end{array}$

$\begin{array}{lll}3.3 & \text { Duality and the physical point } & 13\end{array}$

4 Strength of duality and chirally asymmetric QCD phase diagram $\quad 14$

$\begin{array}{llr}5 & \text { Summary and conclusions } & 18\end{array}$

A Generation of nonzero chiral isospin density $n_{I 5}$ in dense quark matter 20

\section{Introduction}

The phase diagram of strongly interacting matter (QCD phase diagram) is now one of the open questions in the Standard Model of elementary particle physics. The knowledge of QCD phase structure is essential for the understanding of such physical phenomena as compact stars and laboratory experiments on relativistic heavy-ion collisions. While the properties of matter at finite temperatures and vanishing densities are now understood quite well thanks to first principle lattice calculations and heavy-ion collisions, there is no consensus yet on the QCD phase structure at finite chemical potentials. In this case lattice simulations are not possible up to now and most of our understanding comes from calculations performed within effective models. One of the most used models is NambuJona-Lasinio (NJL) model [1, 2] (see for review, e.g., in refs. [3-5]).

Besides the temperature and baryon chemical potential $\mu_{B}$ it is interesting to take into account nonzero isotopic $\mu_{I}$ and chiral chemical potentials. Isotopic (isospin) chemical potential allows us to consider systems with isospin imbalance (different numbers of $u$ and $d$ quarks). Chiral chemical potential $\mu_{5}$ accounts for a chiral imbalance (or density) $n_{5}$ (it is the difference between densities of right-handed and left-handed quarks). It has been argued that the chiral imbalance $n_{5}\left(\right.$ or $\left.\mu_{5}\right)$ can be generated dynamically at high temperatures, for example, in the fireball after heavy ion collision, by virtue of the AdlerBell-Jackiw anomaly and quarks interacting with gauge (gluon) field configurations with nontrivial topology, named sphalerons. On par with an external strong magnetic field, which can be produced in heavy ion collisions as well, this can lead to the so-called chiral magnetic effect [6]. Moreover, in the presence of external magnetic field chiral density $n_{5}$ 
can be produced in dense quark matter due to the so-called chiral separation effect [7]. Similar in essence, the generation mechanism of $n_{5} \neq 0$ can be envisaged also in fast rotating dense matter due to the so-called chiral vortical effect [8]. Chiral imbalance can also be considered as a phenomenon generated within the framework of pure quantum electrodynamics, by coupling quarks to external parallel electric and magnetic fields [9-12]. This mechanism, in our reckoning, is very promising and can lead to a rather large chiral imbalance in the system.

Now let us notice that usually when one talks about chiral density $n_{5}$ (or $\mu_{5}$ ) one implies that separately chiral densities of $u$ and $d$ quarks are equal to each other. Indeed, that is the case when one has in mind the mechanism of generation of chiral imbalance at high temperatures due to nontrivial gauge field configurations. It is obvious due to the fact that gluon field interacts with different quark flavours in exactly the same way and does not feel the difference between flavours. But other mechanisms may be sensitive to the flavour of quarks and we will now elaborate on that. The concept of chiral density (imbalance) can also be introduced for each flavour of quarks. So from now on $n_{5 u}$ and $n_{5 d}$ are the notations for chiral imbalance of $u$ and $d$ quarks, respectively. It is evident that $n_{5}=n_{5 u}+n_{5 d}$ and, correspondingly, $\mu_{5}=\mu_{5 u}+\mu_{5 d}$. But there is a quantity that has not been considered above, namely $n_{I 5}=n_{5 u}-n_{5 d}$ (and, correspondingly, $\mu_{I 5}=\mu_{5 u}-\mu_{5 d}$ ), which is called chiral isospin density (imbalance).

As it has been already noted, the mechanism for generation of the chiral imbalance at high temperatures due to nontrivial gauge field configurations does not work for chiral isospin imbalance, and in this case we have $n_{I 5}=0$. But other mechanisms that were discussed above can lead to its generation, $n_{I 5} \neq 0$. It can be shown that chiral isospin density $n_{I 5}$ can be created in a line with chiral one in dense magnetized quark matter (due to the chiral separation effect) (see in appendix A of the present paper) or in rotating dense quark matter (due to the chiral vortical effect). Moreover, due to the different quark electric charges, in parallel electric and magnetic fields one can get equally successful both chiral and chiral isospin imbalances. This mechanism provides a rather considerable chiral imbalance, both with $n_{5} \neq 0$ and $n_{I 5} \neq 0$, in quark systems, especially in heavy-ion collisions when very strong electric and magnetic fields can be produced in the direction both parallel and perpendicular to the reaction plane $[13,14]$. It probably could be a viable scenario also in neutron stars.

Even though there are several mechanisms of generating chiral isospin imbalance $n_{I 5} \neq 0$, they have not been studied very comprehensively yet and the consideration of chiral isospin imbalance can look academic. But even if one imagines that in physical settings chiral isospin imbalance is absent, still, it could be interesting to study its influence on phase structure of quark matter. Both situations, $\mu_{5} \neq 0$ and $\mu_{I 5} \neq 0$, describe chiral imbalance in the system. If one investigates the influence of chiral imbalance, in general, on some phenomenon for the first time (inhomogeneous phases have not been studied with chiral imbalance of any form) and wants to get some insight, one can first study any of the two types of chiral imbalances that is easier to investigate. In our case one can study the influence of the $n_{I 5} \neq 0\left(\mu_{I 5} \neq 0\right)$ on the inhomogeneous phases just by duality notion (will be discussed below) without performing any numerical calculation (and they are 
rather involved). This example also nicely demonstrates the strength and the beauty of the duality. Of course, since both $\mu_{I 5}$ and $\mu_{5}$ describes chiral imbalance in the system, one can anticipate that the influence of both these imbalances can have similar, at least general, features. For example, both $\mu_{I 5}$ and $\mu_{5}$ generate charged pion condensation (CPC) phase in dense quark matter, although, these generations are various in intensity, or in homogeneous case their influences on chiral symmetry breaking (CSB) are identical at zero baryon and isospin densities [15]. Let us also recall that sometimes it is possible that consideration of unphysical settings can lead to considerable advances in understanding of real physical phenomena. For example, in ref. [16] large isospin density (relative to baryon one) was considered, and it has taught us a great deal about quark pairing and the properties of dense matter.

Moreover, in the context of QCD phase diagram the inclusion of $\mu_{I 5}$ is formally more rigorous than the consideration of $\mu_{5}$. Indeed, the consideration of QCD with chiral imbalance, when $\mu_{5} \neq 0$, does have internal problems, $\mu_{5}$ is not conjugated to a quantity that is strictly conserved in strong interacting matter due to already mentioned Adler-Bell-Jackiw quantum anomaly and chirality changing processes. It can be easily seen in the framework of the NJL model. Chiral charge $n_{5}$ is conjugated to $\mathrm{U}_{A}(1)$ symmetry (which is not conserved due to quantum anomaly) and one can easily see that this symmetry is explicitly broken by NJL model interaction. So the introduction of $\mu_{5}$ is troublesome, although it is massively done in the literature. It is pointed out, however, in ref. [12] that it is possible to consider $\mu_{5}$, if one assumes that the system is observed on a time scale much larger than the typical time scale of the chirality changing processes. Let us note that there is no such problem with chiral isospin imbalance, i.e. when $\mu_{I 5} \neq 0$. In this case in the chiral limit $\mathrm{U}_{I A}(1)$ symmetry (chiral isospin charge $n_{I 5}$ is conjugated to) is anomaly free and conserved in the NJL model.

Recently, it was found a number of strong arguments, corroborated by model calculations, implying that at large and intermediate densities the phases with spatially inhomogeneous order parameters (condensates) can exist in different branches of modern physics. If ground state of a theory is inhomogeneous, then some of its spatial symmetries are spontaneously broken. This phenomenon is not a quite new concept. For example, the idea of density waves in nuclear matter was already discussed in 1960 by Overhauser [17]. Moreover, in the 1970s there was much activity related to inhomogeneous pion condensation $[18,19]$, first considered by Migdal [20, 21]. Spatially inhomogeneous phases in nucleon matter were also studied in [22] in terms of sigma model for nucleon and meson fields. These phases in the form of charge and spin density waves are not exotic in condensed matter physics as well [23]. In particular, the crystalline phases have been considered long time ago for superconductors by Larkin and Ovchinikov [24] as well as by Fulde and Ferrell [25] (usually, the phase is called LOFF phase), etc. And more recently crystalline inhomogeneous phases in color superconducting quark matter were discussed in refs. [26-31]. Deryagin, Grigoriev, and Rubakov (DGR) have shown that at high densities in the limit of infinite number of colors $N_{c}$ the QCD ground state might be inhomogeneous and anisotropic so that the QCD ground state has the structure of a spatial standing wave [32]. And, of course, spatially nonuniform condensates are intensively studied in the framework of ef- 
fective theories such as $\mathrm{NJL}_{4}$ model [33-35] or different (1+1)-dimensional theories with four-fermionic interactions [36-41]. The modern state of investigations of dense baryonic matter in the framework of the spatially inhomogeneous condensate approach is presented in the reviews $[42,43]$.

Unfortunately, general analytical methods, which can be used in order to establish the inhomogeneity of the ground state of an arbitrary system, are absent. So different ansatzes for spatially inhomogeneous condensates are used. For example, for charged pion and/or superconducting condensates usually the single-plane-wave LOFF ansatz is employed [44-46]. The simplest possible spatially inhomogeneous ansatz for chiral condensate in quantum field theories is an analog of the spin-density waves in condensed matter systems, and it is called "chiral density wave" (CDW) [47]. Being an analytically treatable case, CDW ansatz has been the object of intense investigations during the course of the last 25 years and provides us with an excellent prototype for many generic features of inhomogeneous condensation in dense matter (see, e.g., refs. [48-60]). Of course, there can be a more favorable form of the inhomogeneous chiral condensate that minimizes thermodynamic potential even more effectively, but using CDW ansatz we can at least conclude that system favours inhomogeneity in some regions. A more interesting fact is is that recently it was argued in refs. [61, 62] that at small temperatures and rather high baryon densities the so-called Quarkyonic phase can be realized in QCD. This phase is described locally by the chiral condensate also in the form of chiral density waves. Furthermore, some realistic parity doublet hadron models, see, e.g., in refs. [63, 64], predict the CDW phase of dense nuclear matter.

Duality is another interesting possible property of dense matter, which permits to relate different regions of its phase diagram. For example, previously in refs. [46, 65-67] it has been found a duality correspondence between CSB and superconductivity phenomena on the phase diagram of some low-dimensional NJL-type models extended by baryon and chiral $\mu_{5}$ chemical potentials. It means that if inside some definite region of a phase diagram the CSB phase is realized, then in a dually conjugated region the superconductivity must be observed, and vice versa. Note that this kind of duality is based on the invariance of the initial NJL-type Lagrangians both in two- and three-dimensional spacetimes with respect to some permutation of chemical potentials and coupling constants as well as discrete Pauli-Gursey transformations of spinor fields, which connect CSB and superconducting interaction channels (see, e.g., in refs. [46, 67]). We call this additional invariance of the Lagrangian as its dual symmetry. (In contrast, the ordinary Lagrangian symmetry is its invariance with respect to certain field transformations only, when external parameters such as chemical potentials etc. remain intact.) Moreover, just due to the dual symmetry of the Lagrangian it is also possible to assert that if in the system a particular spatially inhomogeneous phase is realized, then dually transformed phase also exists and it is spatially inhomogeneous.

In our previous papers $[15,68-70]$ the phase diagram of dense baryon matter with chiral and isospin imbalances has been considered in the framework of the massless NJL model with two quark flavors (see Lagrangian (2.1) below) both in $(3+1)$ - and $(1+1)$-dimensional spacetime. The model is designed to describe CSB and CPC phenomena of dense matter. Using a homogeneous ansatz for chiral and pion condensates, we have proved in these 
papers that in the large- $N_{c}$ limit, where $N_{c}$ is the number of colors, there is a duality correspondence (symmetry) between CSB and CPC phenomena on the phase diagram of this effective model. However, at $N_{c} \geq 3$ this symmetry of the phase diagram (or thermodynamic potential) does not correspond to any dual symmetry between CSB and CPC channels of the NJL Lagrangian (2.1) (at least, we were unable to find such additional Lagrangian invariance, typical to some low-dimensional models [46, 67], which describes the interaction in CSB and superconducting channels). Hence, (i) this kind of duality can be considered only as a dynamical effect, manifested in the symmetry of the thermodynamic potential (TDP) in the leading $1 / N_{c^{-}}$order of the model with respect to some dual (see below) transformations both of order parameters and chemical potentials. (ii) It is not certain that studies of the phase structure of the model in the next orders over $1 / N_{c}$ will give the same result. It is likely that this duality of the phase portrait of the NJL model (2.1) between CSB and CPC phenomena is only approximate. Finally, (iii) the fact that there is a dual symmetry of the TDP, calculated in the framework of a homogeneous approach to condensates in the leading $1 / N_{c}$-order, does not at all mean that there should be a duality between spatially inhomogeneous CSB and CPC phases. ${ }^{1}$

The proposed work is devoted to the consideration of this last (iii) problem. In our paper isotopically and chirally asymmetric dense baryon matter is investigated, as in ref. [15], in the framework of $(3+1)$-dimensional NJL model. However, in contrast to ref. [15], here we take into account the possibility of spatially inhomogeneous both chiral and charged pion condensates. For the chiral condensate we use the CDW ansatz, while the single-planewave LOFF ansatz is used for the charged pion one. Our main result is the conclusion that in the leading order of the large- $N_{c}$ expansion the $\mathrm{NJL}_{4}$ model predicts the duality between these inhomogeneous phases. The phase structure itself with inhomogeneous condensates is not studied numerically here and it could be the subject of the future work. But the other method of obtaining information of the phase diagram has been used, namely, the studied duality was employed. We note that earlier some aspects of the phase diagram of isotopically asymmetric dense baryon matter have been studied in the framework of inhomogeneous condensate approach, and it turns out that it is possible to combine the findings of these studies together and draw full phase diagram. Then, applying just to this phase diagram the dual mapping, we obtain the full phase diagram of chirally asymmetric dense baryon matter. The obtained phase diagram is quite rich and contains various inhomogeneous phases, both inhomogeneous chiral symmetry breaking phase and inhomogeneous charged pion condensation phase. This example shows that the duality is not just interesting and entertaining mathematical property but a potent instrument with very high predictive capabilities.

The paper is organized as follows. First, in section 2 a $(3+1)$-dimensional massless NJL model with two quark flavors ( $u$ and $d$ quarks) that includes four kinds of chemical

\footnotetext{
${ }^{1}$ Earlier, it was found in the paper [44] that in the framework of the (3+1)-dimensional NJL model (2.1) the spatially inhomogeneous, e.g., CPC phase can be realized in dense isotopically asymmetric matter at some chemical potential region. Then, if we had confidence in the existence of a duality between nonuniform CSB and CPC phases, we could predict the existence of an inhomogeneous CSB phase in the dually conjugated region of chemical potentials.
} 
potentials, $\mu_{B}, \mu_{I}, \mu_{I 5}, \mu_{5}$, is presented. Here spatially inhomogeneous CDW and singleplane-wave LOFF ansatzes respectively for chiral and charged pion condensates are introduced, and the expression for the TDP of the system in the leading $1 / N_{c}$ order is obtained. In section 3 we show that in the homogeneous approach to condensates the TDP is invariant with respect to three dual symmetries. One of them, $\mathcal{D}_{H}(3.3)$, corresponds to the duality of the phase structure of the model between CSB and CPC phenomena. Just this symmetry of the TDP is realized within an inhomogeneous approach. However, two other dual symmetries of the TDP have no analogs in the inhomogeneous approach to the investigation of the ground state of the system. In section 4 we show that using only the duality property of dense quark matter, it is possible, basing on the well-known $\left(\mu_{I}, \mu\right)$-QCD phase diagram, to construct the $\left(\mu_{I 5}, \mu\right)$-phase portrait without any numerical studies.

\section{The model and thermodynamic potential}

We study a phase structure of the two flavored massless $(3+1)$-dimensional NJL model with several chemical potentials. Its Lagrangian, which is symmetrical under global color $\mathrm{SU}\left(N_{c}\right)$ group, has the form

$$
L=\bar{q}\left[\gamma^{\nu} \mathrm{i} \partial_{\nu}+\frac{\mu_{B}}{3} \gamma^{0}+\frac{\mu_{I}}{2} \tau_{3} \gamma^{0}+\mu_{5} \gamma^{0} \gamma^{5}+\frac{\mu_{I 5}}{2} \tau_{3} \gamma^{0} \gamma^{5}\right] q+\frac{G}{N_{c}}\left[(\bar{q} q)^{2}+\left(\bar{q} \mathrm{i} \gamma^{5} \vec{\tau} q\right)^{2}\right]
$$

and describes dense quark matter with two massless $u$ and $d$ quarks, i.e. $q$ in $(2.1)$ is the flavor doublet, $q=\left(q_{u}, q_{d}\right)^{T}$, where $q_{u}$ and $q_{d}$ are four-component Dirac spinors as well as color $N_{c}$-plets (the summation in (2.1) over flavor, color, and spinor indices is implied); $\tau_{k}$ $(k=1,2,3)$ are Pauli matrices. The Lagrangian (2.1) contains baryon $\mu_{B}$, isospin $\mu_{I}$, chiral isospin $\mu_{I 5}$ and chiral $\mu_{5}$ chemical potentials. In other words, this model is able to describe the properties of quark matter with nonzero baryon $n_{B}$, isospin $n_{I}$, chiral isospin $n_{I 5}$ and chiral $n_{5}$ densities, which are the quantities thermodynamically conjugated to chemical potentials $\mu_{B}, \mu_{I}, \mu_{I 5}$ and $\mu_{5}$, respectively. Notice that Lagrangian (2.1) at low energies describes effectively QCD only at $N_{c} \geq 3$. At $N_{c}=2$ QCD is additionally symmetric with respect to the so-called Pauli-Gursey symmetry (at zero chemical potentials) and effectively described by another NJL-type Lagrangian with diquark interaction channel [71].

The quantities $n_{B}, n_{I}$ and $n_{I 5}$ are densities of conserved charges, which correspond to the invariance of Lagrangian (2.1) with respect to the abelian $\mathrm{U}_{B}(1), \mathrm{U}_{I_{3}}(1)$ and $\mathrm{U}_{A I_{3}}(1)$ groups, where ${ }^{2}$

$$
\mathrm{U}_{B}(1): q \rightarrow \exp (\mathrm{i} \alpha / 3) q ; \mathrm{U}_{I_{3}}(1): q \rightarrow \exp \left(\mathrm{i} \alpha \tau_{3} / 2\right) q ; \mathrm{U}_{A I_{3}}(1): q \rightarrow \exp \left(\mathrm{i} \alpha \gamma^{5} \tau_{3} / 2\right) q .
$$

So we have from (2.2) that $n_{B}=\bar{q} \gamma^{0} q / 3, n_{I}=\bar{q} \gamma^{0} \tau^{3} q / 2$ and $n_{I 5}=\bar{q} \gamma^{0} \gamma^{5} \tau^{3} q / 2$. We would like also to remark that, in addition to (2.2), Lagrangian (2.1) is invariant with respect to the electromagnetic $\mathrm{U}_{Q}(1)$ group,

$$
\mathrm{U}_{Q}(1): q \rightarrow \exp (\mathrm{i} Q \alpha) q,
$$

\footnotetext{
${ }^{2}$ Recall for the following that $\exp \left(\mathrm{i} \alpha \tau_{3}\right)=\cos \alpha+\mathrm{i} \tau_{3} \sin \alpha, \exp \left(\mathrm{i} \alpha \gamma^{5} \tau_{3}\right)=\cos \alpha+\mathrm{i} \gamma^{5} \tau_{3} \sin \alpha$.
} 
where $Q=\operatorname{diag}(2 / 3,-1 / 3)$. However, the chiral chemical potential $\mu_{5}$ does not correspond to a conserved quantity of the model (2.1). It is usually introduced in order to describe a system on the time scales when all chirality changing processes are finished in the system and it is in the state of thermodynamical equilibrium with some fixed value of the chiral density $n_{5}$ [72-80]. The ground state expectation values of $n_{B}, n_{I}, n_{I 5}$ and $n_{5}$ can be found by differentiating the thermodynamic potential of the system (2.1) with respect to the corresponding chemical potentials.

In our previous paper [15] it was shown that if an approach with spatially homogeneous condensates is applied to the model (2.1), then thermodynamic potential (TDP) of the system obeys some symmetry, which is manifested in the duality between CSB and CPC phenomena of dense quark matter (see below). The goal of the present paper is to show that this duality must also manifest itself in the approach with inhomogeneous condensates. At the same time, for chiral and charged pion condensates we use the CDW and singleplane-wave LOFF ansatzes, respectively.

Let us note that the ansatz used in our present paper includes several possible inhomogeneous condensates but one may wonder why we do not reproduce the inhomogeneous condensate analyzed by DGR at high baryon densities. Indeed, in [32] finitedensity QCD in the large $N_{c}$ limit has been discussed and it has been noticed that at high baryon densities Fermi surface is unstable with respect to the formation of chiral waves with wavenumber $2 p_{F}$, where $p_{F}$ is the Fermi momentum. But they worked in the perturbative regime $\mathrm{g}^{2} N_{c} \ll 1$. In [81, 82], where it was analyzed what happens to the DGR instability at large but finite $N_{c}$, the region of the DGR phase was restricted by the line $\mu>3 \Lambda_{\mathrm{QCD}} \sim 650-700 \mathrm{MeV}$, where $\mu=\mu_{B} / 3$ is the quark number chemical potential (maybe in reality, it should be much higher), because below this line the QCD system is certainly a strongly-coupled and there perturbative calculations cannot be trusted. As a rule, perturbative QCD (without large $N_{c}$ limit) starts to work at very high values of chemical potential, $\mu \gg 1 \mathrm{GeV}$ [83]. But at that large values of chemical potentials the NJL model is not expected to give trustworthy results, as it is an effective low-energy model for QCD, which is adequate only up to the cut-off, which is around $650 \mathrm{MeV}$. And the region of the phase diagram with values of the larger $\mu$ is out of scope of the consideration. Let us also notice that at large values of $\mu$, even not necessarily as large as $650-700 \mathrm{MeV}$, the system is probably in the color superconducting phase. DGR noticed that color superconductivity is suppressed at large $N_{c}$ due to the fact that the Cooper pair is not a color singlet (the diagram responsible for color superconductivity is non-planar) and only in the large $N_{c}$ limit the DGR phase is predicted. So summarizing, DGR is an asymptotic phase (at high $\mu$ ) and cannot be obtained at low values of $\mu$, whereas NJL model describe only the region of comparatively low values of $\mu$.

To find the TDP of the system, we use a semibosonized version of the Lagrangian (2.1), which contains composite bosonic fields $\sigma(x)$ and $\pi_{a}(x)(a=1,2,3)$ (in what follows, we use the notations $\mu \equiv \mu_{B} / 3, \nu \equiv \mu_{I} / 2$ and $\left.\nu_{5} \equiv \mu_{I 5} / 2\right)$ :

$$
\widetilde{L}=\bar{q}\left[\gamma^{\rho} \mathrm{i} \partial_{\rho}+\mu \gamma^{0}+\nu \tau_{3} \gamma^{0}+\mu_{5} \gamma^{0} \gamma^{5}+\nu_{5} \tau_{3} \gamma^{0} \gamma^{5}-\sigma-\mathrm{i} \gamma^{5} \pi_{a} \tau_{a}\right] q-\frac{N_{c}}{4 G}\left[\sigma \sigma+\pi_{a} \pi_{a}\right] .
$$


In (2.4) and below the summation over repeated indices is implied. From the auxiliary Lagrangian (2.4) one gets the equations for the bosonic fields

$$
\sigma(x)=-2 \frac{G}{N_{c}}(\bar{q} q) ; \quad \pi_{a}(x)=-2 \frac{G}{N_{c}}\left(\bar{q} \mathrm{i} \gamma^{5} \tau_{a} q\right) .
$$

Note that the composite bosonic field $\pi_{3}(x)$ can be identified with the physical $\pi^{0}(x)$ meson field, whereas the physical $\pi^{ \pm}(x)$-meson fields are the following combinations of the composite fields, $\pi^{ \pm}(x)=\left(\pi_{1}(x) \mp i \pi_{2}(x)\right) / \sqrt{2}$. Obviously, the semibosonized Lagrangian $\widetilde{L}$ is equivalent to the initial Lagrangian (2.1) when using the equations (2.5). Furthermore, it is clear from (2.2) and footnote 2 that the composite bosonic fields (2.5) are transformed under the isospin $\mathrm{U}_{I_{3}}(1)$ and axial isospin $\mathrm{U}_{A I_{3}}(1)$ groups in the following manner:

$$
\begin{array}{rcccc}
\mathrm{U}_{I_{3}}(1): & \sigma \rightarrow \sigma ; & \pi_{3} \rightarrow \pi_{3} ; & \pi_{1} \rightarrow \cos (\alpha) \pi_{1}+\sin (\alpha) \pi_{2} ; & \pi_{2} \rightarrow \cos (\alpha) \pi_{2}-\sin (\alpha) \pi_{1}, \\
\mathrm{U}_{A I_{3}}(1): & \pi_{1} \rightarrow \pi_{1} ; & \pi_{2} \rightarrow \pi_{2} ; & \sigma \rightarrow \cos (\alpha) \sigma+\sin (\alpha) \pi_{3} ; & \pi_{3} \rightarrow \cos (\alpha) \pi_{3}-\sin (\alpha) \sigma .
\end{array}
$$

Starting from the theory (2.4), one obtains in the leading order of the large $N_{c}$-expansion (i.e. in the one-fermion loop approximation) the following path integral expression for the effective action $\mathcal{S}_{\text {eff }}\left(\sigma, \pi_{a}\right)$ of the bosonic $\sigma(x)$ and $\pi_{a}(x)$ fields:

$$
\exp \left(\mathrm{i} \mathcal{S}_{\text {eff }}\left(\sigma, \pi_{a}\right)\right)=N^{\prime} \int[d \bar{q}][d q] \exp \left(\mathrm{i} \int \widetilde{L} d^{4} x\right)
$$

where

$$
\mathcal{S}_{\text {eff }}\left(\sigma, \pi_{a}\right)=-N_{c} \int d^{4} x\left[\frac{\sigma^{2}+\pi_{a}^{2}}{4 G}\right]+\tilde{\mathcal{S}}_{\text {eff }}
$$

and $N^{\prime}$ is a normalization constant. The quark contribution to the effective action, i.e. the term $\tilde{\mathcal{S}}_{\text {eff }}$ in eq. (2.7), is given by:

$$
\exp \left(\mathrm{i} \tilde{\mathcal{S}}_{\text {eff }}\right)=N^{\prime} \int[d \bar{q}][d q] \exp \left(\mathrm{i} \int\left\{\bar{q}\left[\gamma^{\rho} \mathrm{i} \partial_{\rho}+\mu \gamma^{0}+\nu \tau_{3} \gamma^{0}+\mu_{5} \gamma^{0} \gamma^{5}+\nu_{5} \tau_{3} \gamma^{0} \gamma^{5}-\sigma-\mathrm{i} \gamma^{5} \pi_{a} \tau_{a}\right] q\right\} d^{4} x\right) .
$$

The ground state expectation values $\langle\sigma(x)\rangle$ and $\left\langle\pi_{a}(x)\right\rangle$ of the composite bosonic fields are determined by the saddle point equations,

$$
\frac{\delta \mathcal{S}_{\mathrm{eff}}}{\delta \sigma(x)}=0, \quad \frac{\delta \mathcal{S}_{\mathrm{eff}}}{\delta \pi_{a}(x)}=0,
$$

where $a=1,2,3$. It is clear from eq. (2.6) that if $\langle\sigma(x)\rangle \neq 0$ and/or $\left\langle\pi_{3}(x)\right\rangle \neq 0$, then the axial isospin $\mathrm{U}_{A I_{3}}(1)$ symmetry of the model is spontaneously broken down, whereas at $\left\langle\pi_{1}(x)\right\rangle \neq 0$ and/or $\left\langle\pi_{2}(x)\right\rangle \neq 0$ we have a spontaneous breaking of the isospin $\mathrm{U}_{I_{3}}(1)$ symmetry. Since in the latter case the ground state expectation values, or condensates, both of the field $\pi^{+}(x)$ and of the field $\pi^{-}(x)$ are nonzero, this phase is usually called the CPC phase. It is easy to see from eq. (2.5) that the nonzero condensates $\left\langle\pi_{1,2}(x)\right\rangle$ (or $\left.\left\langle\pi^{ \pm}(x)\right\rangle\right)$ are not invariant with respect to the electromagnetic $\mathrm{U}_{Q}(1)$ transformations $(2.3)$ of the flavor quark doublet. Hence in the CPC phase the electromagnetic $\mathrm{U}_{Q}(1)$ invariance 
of the model (2.1) is also broken spontaneously, so superconductivity is an unavoidable property of the CPC phase.

In vacuum, i.e. in the state corresponding to an empty space with zero particle density and zero values of the chemical potentials $\mu, \nu, \mu_{5}$ and $\nu_{5}$, the quantities $\langle\sigma(x)\rangle$ and $\left\langle\pi_{a}(x)\right\rangle$ do not depend on space coordinate $x$. However, in dense medium, when some of the chemical potentials are nonzero quantities, the ground state expectation values of bosonic fields might have a nontrivial dependence on spatial coordinates. In particular, in this paper we use the following spatially inhomogeneous CDW ansatz for chiral condensate and the singleplane-wave LOFF ansatz for charged pion condensates (for simplicity we suppose that wavevectors of the inhomogeneous condensates are directed along the $x^{1}$ coordinate axis):

$$
\begin{aligned}
& \langle\sigma(x)\rangle=M \cos \left(2 k x^{1}\right), \quad\left\langle\pi_{3}(x)\right\rangle=M \sin \left(2 k x^{1}\right), \\
& \left\langle\pi_{1}(x)\right\rangle=\Delta \cos \left(2 k^{\prime} x^{1}\right), \quad\left\langle\pi_{2}(x)\right\rangle=\Delta \sin \left(2 k^{\prime} x^{1}\right),
\end{aligned}
$$

where gaps $M, \Delta$ and wavevectors $k, k^{\prime}$ are constant dynamical quantities. In fact, they are coordinates of the global minimum point of the TDP $\Omega\left(M, k, k^{\prime}, \Delta\right)$. In the leading order of the large- $N_{c}$ expansion it is defined by the following expression:

$$
\int d^{4} x \Omega\left(M, k, k^{\prime}, \Delta\right)=-\left.\frac{1}{N_{c}} \mathcal{S}_{\mathrm{eff}}\left\{\sigma(x), \pi_{a}(x)\right\}\right|_{\sigma(x)=\langle\sigma(x)\rangle, \pi_{a}(x)=\left\langle\pi_{a}(x)\right\rangle},
$$

which gives

$$
\int d^{4} x \Omega\left(M, k, k^{\prime}, \Delta\right)=\int d^{4} x \frac{M^{2}+\Delta^{2}}{4 G}+\frac{\mathrm{i}}{N_{c}} \ln \left(\int[d \bar{q}][d q] \exp \left(\mathrm{i} \int d^{4} x \bar{q} \widetilde{D} q\right)\right),
$$

where

$$
\begin{aligned}
\bar{q} \widetilde{D} q= & \bar{q}\left[\gamma^{\rho} \mathrm{i} \partial_{\rho}+\mu \gamma^{0}+\nu \tau_{3} \gamma^{0}+\mu_{5} \gamma^{0} \gamma^{5}+\nu_{5} \tau_{3} \gamma^{0} \gamma^{5}-M \exp \left(2 \mathrm{i} \gamma^{5} \tau_{3} k x^{1}\right)\right] q \\
& -\Delta\left(\bar{q}_{u} \mathrm{i} \gamma^{5} q_{d}\right) \mathrm{e}^{-2 \mathrm{i} k^{\prime} x^{1}}-\Delta\left(\bar{q}_{d} \mathrm{i} \gamma^{5} q_{u}\right) \mathrm{e}^{2 \mathrm{i} k^{\prime} x^{1}} .
\end{aligned}
$$

(Remember that in this formula $q$ is indeed a flavor doublet, i.e. $q=\left(q_{u}, q_{d}\right)^{T}$.) To proceed, let us introduce in eqs. (2.12)-(2.13) the new quark doublets, $\psi$ and $\bar{\psi}$, by the so-called Weinberg (or chiral) transformation of these fields [69, 84], $\psi=\exp \left(\mathrm{i} \tau_{3} k^{\prime} x^{1}+\mathrm{i} \tau_{3} \gamma^{5} k x^{1}\right) q$ and $\bar{\psi}=\bar{q} \exp \left(\mathrm{i} \tau_{3} \gamma^{5} k x^{1}-\mathrm{i} \tau_{3} k^{\prime} x^{1}\right)$. Since this transformation of quark fields does not change the path integral measure in eq. $(2.12),{ }^{3}$ the expression (2.12) for the TDP is easily transformed to the following one:

$$
\int d^{4} x \Omega\left(M, k, k^{\prime}, \Delta\right)=\int d^{4} x \frac{M^{2}+\Delta^{2}}{4 G}+\frac{\mathrm{i}}{N_{c}} \ln \left(\int[d \bar{\psi}][d \psi] \exp \left(\mathrm{i} \int d^{4} x \bar{\psi} D \psi\right)\right),
$$

\footnotetext{
${ }^{3}$ Strictly speaking, performing Weinberg transformation of quark fields in eq. (2.12), one can obtain in the path integral measure a factor, which however does not depend on the dynamical variables $M, \Delta, k$, and $k^{\prime}$. Hence, we ignore this unessential factor in the following calculations. Note that only in the case when there is an interaction between spinor and gauge fields there might appear a nontrivial, i.e. dependent on dynamical variables, path integral measure, generated by Weinberg transformation of spinors. This unobvious fact follows from the investigations by Fujikawa [85].
} 
where instead of the $x$-dependent Dirac operator $\widetilde{D}$ a new $x$-independent operator $D=i \gamma^{\mu} \partial_{\mu}+\mu \gamma^{0}+\nu \tau_{3} \gamma^{0}+\mu_{5} \gamma^{0} \gamma^{5}+\nu_{5} \tau_{3} \gamma^{0} \gamma^{5}+\tau_{3} \gamma^{1} \gamma^{5} k+\tau_{3} \gamma^{1} k^{\prime}-M-\mathrm{i} \gamma^{5} \Delta \tau_{1}$ appears. In this case path integral can be evaluated and one get for the TDP (2.12) an expression that reads

$$
\Omega\left(M, \Delta, k, k^{\prime}\right)=\frac{M^{2}+\Delta^{2}}{4 G}+\mathrm{i} \frac{\operatorname{Tr}_{s f x} \ln D}{\int d^{4} x}=\frac{M^{2}+\Delta^{2}}{4 G}+\mathrm{i} \int \frac{d^{4} p}{(2 \pi)^{4}} \ln \operatorname{Det} \bar{D}(p),
$$

where

$\bar{D}(p)=\not p+\mu \gamma^{0}+\nu \tau_{3} \gamma^{0}+\mu_{5} \gamma^{0} \gamma^{5}+\nu_{5} \tau_{3} \gamma^{0} \gamma^{5}+\tau_{3} \gamma^{1} \gamma^{5} k+\tau_{3} \gamma^{1} k^{\prime}-M-\mathrm{i} \gamma^{5} \Delta \tau_{1} \equiv\left(\begin{array}{c}A, U \\ V, B\end{array}\right)$

is the momentum space representation of the Dirac operator $D$. The quantities $A, B, U, V$ in (2.16) are really the following $4 \times 4$ matrices in the spinor space,

$$
\begin{aligned}
A & =\not p+\mu \gamma^{0}+\nu \gamma^{0}+\mu_{5} \gamma^{0} \gamma^{5}+\nu_{5} \gamma^{0} \gamma^{5}-M+\gamma^{1} \gamma^{5} k+\gamma^{1} k^{\prime} ; \\
B & =\not p+\mu \gamma^{0}-\nu \gamma^{0}+\mu_{5} \gamma^{0} \gamma^{5}-\nu_{5} \gamma^{0} \gamma^{5}-M-\gamma^{1} \gamma^{5} k-\gamma^{1} k^{\prime} ; \\
U=V & =-\mathrm{i} \gamma^{5} \Delta,
\end{aligned}
$$

so the quantity $\bar{D}(p)$ from (2.16) is indeed a $8 \times 8$ matrix whose determinant in eq. (2.15) can be calculated on the basis of the following general relations

$\operatorname{Det} \bar{D}(p) \equiv \operatorname{det}\left(\begin{array}{l}A, U \\ V, B\end{array}\right)=\operatorname{det}\left[-V U+V A V^{-1} B\right]=\operatorname{det}\left[\Delta^{2}+\gamma^{5} A \gamma^{5} B\right]=\operatorname{det}\left[B A-B U B^{-1} V\right]$.

\section{Duality between CSB and CPC phenomena}

\subsection{Spatially homogeneous approach to condensates}

In this case the wave vectors $k$ and $k^{\prime}$ in the inhomogeneous ansatzes (2.10) are zero by assumption and, as a result, for the quantity (2.18) one can get the following expression (for details see ref. [15])

$$
\operatorname{Det} \bar{D}(p)=\left(\eta^{4}-2 a_{+} \eta^{2}+b_{+} \eta+c_{+}\right)\left(\eta^{4}-2 a_{-} \eta^{2}+b_{-} \eta+c_{-}\right) \equiv P_{+}\left(p_{0}\right) P_{-}\left(p_{0}\right),
$$

where $\eta=p_{0}+\mu,|\vec{p}|=\sqrt{p_{1}^{2}+p_{2}^{2}+p_{3}^{2}}$ and

$$
\begin{aligned}
& a_{ \pm}=M^{2}+\Delta^{2}+\left(|\vec{p}| \pm \mu_{5}\right)^{2}+\nu^{2}+\nu_{5}^{2} ; \quad b_{ \pm}= \pm 8\left(|\vec{p}| \pm \mu_{5}\right) \nu \nu_{5} \\
& c_{ \pm}=a_{ \pm}^{2}-4 \nu^{2}\left(M^{2}+\left(|\vec{p}| \pm \mu_{5}\right)^{2}\right)-4 \nu_{5}^{2}\left(\Delta^{2}+\left(|\vec{p}| \pm \mu_{5}\right)^{2}\right)-4 \nu^{2} \nu_{5}^{2} .
\end{aligned}
$$

One can notice that in this case the expression (3.1) and hence the TDP (2.15) are invariant with respect to the so-called duality transformation $\mathcal{D}_{H}$ of the order parameters and chemical potentials,

$$
\mathcal{D}_{H}: \quad M \longleftrightarrow \Delta, \quad \nu \longleftrightarrow \nu_{5} .
$$


Other parameters such as $\mu, \mu_{5}$ and the coupling constant $G$ do not change under this transformation. It means that if at $\mu, \mu_{5}, \nu=P, \nu_{5}=Q$ the global minimum of the TDP lies at the point $\left(M=M_{0}, \Delta=\Delta_{0}\right)$, then at $\mu, \mu_{5}, \nu=Q, \nu_{5}=P$ it is at the point $\left(M=\Delta_{0}, \Delta=M_{0}\right)$. The results of the paper [15] support this conclusion. In particular, it was shown there that if at some fixed values of chemical potentials, e.g., the homogeneous CSB phase is realized, then in the dually conjugated region of the chemical potentials, i.e. at $\nu \leftrightarrow \nu_{5}$ and unchanged values of $\mu$ and $\mu_{5}$, the homogeneous CPC phase must be observed in the system and vice versa.

The duality similar to eq. (3.3), i.e. the duality between CSB and CPC phenomena, is also observed between phase structures of gauge theories with different gauge groups and matter content in the framework of the so-called orbifold equivalence formalism in the large- $N_{c}$ limit $[86,87]$.

In addition to invariance of the TDP with respect to the duality transformation (3.3), in the case of homogeneous approach to the condensates there are two other transformations of chemical potentials and order parameters, $\mathcal{D}_{H M}$ and $\mathcal{D}_{H \Delta}$, which we call constrained dual transformations, that leave the TDP unchanged. Indeed, one can check (see in ref. [15]) that under the constraint $\Delta=0$ and at fixed values of $\mu, \nu$ the $\operatorname{TDP}(2.15)$ at $k, k^{\prime}=0$ is invariant with respect to the permutation $\mu_{5} \leftrightarrow \nu_{5}$. It is the so-called constrained dual transformation $\mathcal{D}_{H M}$. Whereas at $M=0$ and at fixed values of $\mu, \nu_{5}$ the TDP (2.15) at $k, k^{\prime}=0$ is invariant under the permutation $\mu_{5} \leftrightarrow \nu$. It is the so-called constrained dual transformation $\mathcal{D}_{H \Delta}$. The symmetry of the TDP with respect to $\mathcal{D}_{H M}$ (with respect to $\mathcal{D}_{H \Delta}$ ) means that if at some values of the chemical potentials the CSB phase (the CPC phase) is realized, then at $\mu_{5} \leftrightarrow \nu_{5}$ (at $\mu_{5} \leftrightarrow \nu$ ) the same phase will be observed, if dynamically or due to other reasons the charged pion condensate $\Delta$ is equal to zero (the chiral condensate $M$ is equal to zero) in the system. Hence, in the case of a homogeneous approach to condensates the symmetry of the TDP under the constrained $\mathcal{D}_{H M}$ and $\mathcal{D}_{H \Delta}$ dual transformations can also be useful in relating phase structure of the model between dually conjugated regions of the chemical potentials.

Of course, the duality between CSB and CPC phenomena can be broken by expectation values of operators that have not been included into the consideration. For example, on the QCD phase diagram there could be more complicated light meson condensations, such as $\rho-, \omega$ - meson or kaon, etc. that are not considered in our paper and, in principle, can break the duality of the phase structure. Let us make a couple of comments and show that it is likely that all or a massive region of phase diagram considered in the paper does not have these condensates and, hence, these condensations can be excluded from the consideration. It was suggested early on in [88-90] that at sufficiently high $\mu_{I}$, charged $\rho$-mesons will undergo Bose-Einstein condensation as pions. But it has been concluded in $[91,92]$ that $\rho$-meson condensation is possible only at isospin chemical potentials much higher than the $\rho$ meson mass and, in the context of our consideration, this is outside of the range that we are interested in (range of validity of NJL model). There is another possibility such as $\omega$-meson condensation (which is equivalent to inclusion of vector interaction) but, it effectively shifts baryon chemical potential $\mu_{B}$ and does not spoil the duality. Kaon condensation cannot be considered in two-flavoured NJL model at all. So in our studies we take out of consideration 
possibility of all the light meson condensations except the pion one. Also, there is a possibility of colour superconducting phase. If there exist non-zero diquark condensates, then the duality, in general, can be spoiled as well. But here in our considerations we assumed that it is zero, let us elaborate on that. Color superconductivity arises (in most of the approaches) for chemical potential $\mu$ larger than approximately $350 \mathrm{MeV}$. Maybe, it is possible to say that the color superconductivity is not likely realized below $350 \mathrm{MeV}$ and, additionally, it is shown in [77] that if one includes $\mu_{5}$ into consideration the transition to the color superconducting phase shifts to higher values of $\mu$. Nevertheless, all these arguments do not forbid color superconductivity and we should admit that above certain value of $\mu$ we neglected a gap from Cooper pairing just for simplicity.

\subsection{Duality in inhomogeneous case}

Let us now discuss the possibility of the duality between CSB and CPC phenomena when spatially inhomogeneous approach in the form (2.10) to condensates is used, i.e. we suppose that $k \neq 0, k^{\prime} \neq 0$. In this case, using any program of analytic calculations, it is also possible to obtain an exact analytical expression for the quantity (2.18) in the form of the 8-th order polynomial,

$$
\operatorname{Det} \bar{D}(p)=a_{8} \eta^{8}+a_{7} \eta^{7}+a_{6} \eta^{6}+a_{5} \eta^{5}+a_{4} \eta^{4}+a_{3} \eta^{3}+a_{2} \eta^{2}+a_{1} \eta+a_{0}
$$

where $\eta=p_{0}+\mu, a_{8}=1, a_{7}=0$ and

$$
\begin{aligned}
a_{6}= & -4\left(k^{\prime 2}+k^{2}+\Delta^{2}+\nu^{2}+M^{2}+\mu_{5}^{2}+\nu_{5}^{2}+|\vec{p}|^{2}\right), a_{5}=16\left(k^{\prime} k \mu_{5}+\mu_{5} \nu \nu_{5}-\left(k^{\prime} \nu+k \nu_{5}\right) p_{1}\right), \\
a_{4}= & 2\left\{3 k^{\prime 4}+3 k^{4}+2 k^{2} k^{\prime 2}+2 k^{\prime 2}\left(\Delta^{2}+\nu^{2}+3 M^{2}+\mu_{5}^{2}+\nu_{5}^{2}+p_{1}^{2}+3 p_{2}^{2}+3 p_{3}^{2}\right)+\right. \\
& 2 k^{2}\left(3 \Delta^{2}+\nu^{2}+M^{2}+\mu_{5}^{2}+\nu_{5}^{2}+p_{1}^{2}+3 p_{2}^{2}+3 p_{3}^{2}\right)+3\left(\Delta^{2}+M^{2}\right)^{2}+3 \nu^{4}+3 \nu_{5}^{4} \\
& \left.+6\left(M^{2}+\Delta^{2}\right)|\vec{p}|^{2}+3 \mu_{5}^{4}+3|\vec{p}|^{4}+2 \mu_{5}^{2}|\vec{p}|^{2}\right\}+6 \Delta^{2} \nu^{2}+6 M^{2} \nu_{5}^{2}+ \\
& 24 k \nu\left(\mu_{5} p_{1}-k^{\prime} \nu_{5}\right)+24 k^{\prime} \nu_{5}\left(\mu_{5} p_{1}-k \nu\right)+24 \mu_{5} p_{1}\left(k^{\prime} \nu_{5}+k \nu\right)+ \\
& 2 \Delta^{2} \nu_{5}^{2}+2 M^{2} \nu^{2}+2\left(\nu^{2}+\nu_{5}^{2}\right) \mu_{5}^{2}+12 \mu_{5}^{2}\left(\Delta^{2}+M^{2}\right)+2\left(\nu^{2}+\nu_{5}^{2}\right)|\vec{p}|^{2}+ \\
& 2 \nu^{2}\left(3 \Delta^{2}+M^{2}+\mu_{5}^{2}+\nu_{5}^{2}+|\vec{p}|^{2}\right)+2 \nu_{5}^{2}\left(\Delta^{2}+\nu^{2}+3 M^{2}+\mu_{5}^{2}+|\vec{p}|^{2}\right), \\
a_{3}= & 32\left\{k^{\prime 3}\left(\nu p_{1}-k \mu_{5}\right)+k^{3}\left(\nu_{5} p_{1}-k^{\prime} \nu_{5}\right)+k^{\prime 2}\left(\nu \mu_{5} \nu_{5}-k \nu_{5} p_{1}\right)+k^{2}\left(\nu \mu_{5} \nu_{5}-k^{\prime} \nu p_{1}\right)-\right. \\
& k^{\prime} k \mu_{5}\left(\Delta^{2}-\nu^{2}+M^{2}+\mu_{5}^{2}-\nu_{5}^{2}-p_{1}^{2}+p_{2}^{2}+p_{3}^{2}\right)-\nu \mu_{5} \nu_{5}\left(\Delta^{2}+\nu^{2}+M^{2}+\mu_{5}^{2}+\nu_{5}^{2}-|\vec{p}|^{2}\right) \\
& \left.+k^{\prime} \nu p_{1}\left(\Delta^{2}+\nu^{2}+M^{2}-\mu_{5}^{2}-\nu_{5}^{2}+|\vec{p}|^{2}\right)+k \nu_{5} p_{1}\left(\Delta^{2}-\nu^{2}+M^{2}-\mu_{5}^{2}+\nu_{5}^{2}+|\vec{p}|^{2}\right)\right\}, \ldots
\end{aligned}
$$

We do not give here exact analytical expressions for the coefficients $a_{0,1,2}$ of the polynomial (3.4), since they are too extensive and take up too much space. Nevertheless, it is possible to check that all the coefficients $a_{i}$ of the polynomial (3.4) are invariant with respect to the following duality transformation $\mathcal{D}_{I}$ of the chemical potentials, absolute values $\Delta, M$ and wavevectors $k, k^{\prime}$ of the condensates (2.10)

$$
\mathcal{D}_{I}: \quad M \longleftrightarrow \Delta, \quad \nu \longleftrightarrow \nu_{5}, \quad k \longleftrightarrow k^{\prime}
$$


(The invariance of $a_{3,4,5,6}$ with respect to the dual transformation (3.8) is directly seen from eqs. (3.5)-(3.7).) As a result one can find that the whole $\operatorname{TDP} \Omega\left(M, \Delta, k, k^{\prime}\right)(2.15)$ of the system is also invariant under the dual transformation $\mathcal{D}_{I}$. It means that in the leading order of the large- $N_{c}$ approximation the duality correspondence between CSB and CPC phenomena, found in [15] for homogeneous case, is valid even in the case if in the system the phases with spatially inhomogeneous condensates are realized. The dual invariance (3.8) of the TDP allows one to perform also a dual mapping of some well-known QCD phase diagrams in order to predict a phase structure of the system under influence of more exotic external conditions, such as chiral asymmetry, etc (see below in section 4).

In contrast, in the framework of an inhomogeneous approach to condensates in the form (2.10), we were unable to find analogues of two other dual symmetries, the constrained $\mathcal{D}_{H \Delta}$ and $\mathcal{D}_{H M}$ symmetries, which are inherent in the model under consideration within a spatially homogeneous approach to condensates (see in ref. [15] and/or the end of the previous subsection 3.1). Indeed, assuming that in eqs. (3.5)-(3.7) $\Delta=0$ and $k^{\prime}=0$ (or $M=0$ and $k=0$ ), we see that these coefficients of the polynomial (3.4) are not invariant with respect to transposition $\nu_{5} \leftrightarrow \mu_{5}$ (or $\nu \leftrightarrow \mu_{5}$ ). Hence, the analogue of the dual symmetry $\mathcal{D}_{H M}$ (or dual symmetry $\mathcal{D}_{H \Delta}$ ) of the TDP in the homogeneous case is not realized in the case when an inhomogeneous approach to condensates is used. (In fact, in the case of inhomogeneous condensates it is necessary to use more subtle arguments. Indeed, in this approach there arise usually some spurious (unphysical) terms in the TDP (2.15). So it cannot be considered as a physical TDP of the system. To overcome this difficulty, one should use a more physical regularization procedure or apply to TDP the subtraction procedure using the rule presented, e.g., in ref. [69] (see there eqs. (47)-(48)). According to it, we can construct the physical TDP $\Omega^{\text {phys }}\left(M, \Delta, k, k^{\prime}\right)$. It turns out that $\Omega^{\text {phys }}$ is invariant under the dual transformation $\mathcal{D}_{I}$ (3.8). However, both $\Omega^{\text {phys }}\left(M, \Delta=0, k, k^{\prime}=0\right)$ and $\Omega^{\text {phys }}\left(M=0, \Delta, k=0, k^{\prime}\right)$ are not invariant with respect to the transpositions $\nu_{5} \leftrightarrow \mu_{5}$ and $\nu \leftrightarrow \mu_{5}$, correspondingly, i.e. the constrained dual symmetries are not the properties of the TDP in the case of spatially inhomogeneous approach to condensates.)

\subsection{Duality and the physical point}

Though the chiral limit is an excellent approximation to QCD, one knows that in reality the current quark masses are nonzero. Let us now consider briefly the situation with nonzero current quark mass $m_{0}$ (physical point). The way that one can deal with CDW and/or single plane wave ansatz for charged pion condensate at the physical point is the same as in [50]. In this case the Lagrangian looks like

$$
L=\bar{q}\left[\gamma^{\nu} \mathrm{i} \partial_{\nu}+\frac{\mu_{B}}{3} \gamma^{0}+\frac{\mu_{I}}{2} \tau_{3} \gamma^{0}+\mu_{5} \gamma^{0} \gamma^{5}+\frac{\mu_{I 5}}{2} \tau_{3} \gamma^{0} \gamma^{5}-m_{0}\right] q+\frac{G}{N_{c}}\left[(\bar{q} q)^{2}+\left(\bar{q} \mathrm{i} \gamma^{5} \vec{\tau} q\right)^{2}\right]
$$

If current quark mass $m_{0} \neq 0$, then the ansatz (2.10) should be transformed to the following one

$$
\begin{aligned}
& \langle\sigma(x)\rangle=M \cos \left(2 k x^{1}\right)-m_{0}, \quad\left\langle\pi_{3}(x)\right\rangle=M \sin \left(2 k x^{1}\right), \\
& \left\langle\pi_{1}(x)\right\rangle=\Delta \cos \left(2 k^{\prime} x^{1}\right), \quad\left\langle\pi_{2}(x)\right\rangle=\Delta \sin \left(2 k^{\prime} x^{1}\right),
\end{aligned}
$$


where gaps $M, \Delta$ and wavevectors $k, k^{\prime}$ are the same quantities. Using this ansatz in the definition (2.11), one can obtain, instead of eq. (2.14), the following expression for the $\operatorname{TDP} \Omega\left(M, k, k^{\prime}, \Delta\right)$ in the leading large- $N_{c}$ order

$$
\int d^{4} x \Omega\left(M, \Delta, k, k^{\prime}\right)=\int d^{4} x \frac{M^{2}-2 m_{0} M \cos \left(2 k x^{1}\right)+m_{0}^{2}+\Delta^{2}}{4 G}+\operatorname{iTr}_{s f x} \ln D,
$$

where $D$ is the same $x$-independent Dirac operator as in eq. (2.14). The averaging over spacetime coordinates in eq. (3.11) supposes that $\int d^{4} x \cos \left(2 k x^{1}\right)=0$ if $k \neq 0$ and $\int d^{4} x \cos \left(2 k x^{1}\right)=\int d^{4} x$ if $k=0$. So, similar to the ref. [50], we get

$$
\Omega\left(M, \Delta, k, k^{\prime}\right)=\frac{M^{2}-2 m_{0} M \delta_{k, 0}+m_{0}^{2}+\Delta^{2}}{4 G}+\mathrm{i} \int \frac{d^{4} p}{(2 \pi)^{4}} \ln \operatorname{Det} \bar{D}(p),
$$

where $\bar{D}(p)$ is the momentum space representation of the Dirac operator $D$ (see in eq. (2.16)). One can see that the only difference compared to the case of zero current quark mass is one term that is proportional to delta symbol. Hence, if the chiral condensate is homogeneous, then it is easy to see that the duality $\mathcal{D}_{H}$ is no longer the exact symmetry of the TDP, but still one can show that it is a rather good approximation. ${ }^{4}$ But if duality between inhomogeneous phases is concerned, the duality is exact even in the case of nonzero current quark mass $m_{0}$, which is rather interesting in itself.

\section{Strength of duality and chirally asymmetric QCD phase diagram}

Recall that chiral asymmetry is a relatively recent phenomenon, so the QCD phase diagram under this condition has not been studied in detail so far. But investigations of the QCDphase structure at $\mu_{5}, \mu_{I 5}=0$ were performed in the presence of its isospin asymmetry, i.e. at $\mu_{I} \equiv 2 \nu \neq 0$. Let us discuss how it is possible to obtain information about the phase structure of chirally asymmetric dense quark matter (at $\mu_{5} \neq 0$ and/or $\mu_{I 5} \equiv 2 \nu_{5} \neq 0$ ), applying the so-called duality mapping procedure to the well-known QCD-phase diagram with zero chiral asymmetry (at $\mu_{5}=0$ and $\nu_{5}=0$ ).

First, let us discuss what one knows about the QCD $(\nu, \mu)$-phase diagram (dense quark matter with isospin asymmetry). In the framework of a homogeneous ansatz for condensates the QCD phase diagram has been studied in many approaches and models and can be described schematically in the following way (see, e.g., the phase diagram of figure 3 in the paper [44]). If the values of isospin chemical potential $\mu_{I}$ is larger than pion mass $m_{\pi}$, i.e. at $\nu>m_{\pi} / 2$, there is homogeneous CPC phase at rather small values of $\mu$. But if $\mu_{I}$ is less than pion mass, $\nu<m_{\pi} / 2$, then homogeneous CSB phase with zero baryon density (it is the so-called vacuum state) is realized for rather small $\mu$. But for rather large $\mu$ the normal quark matter (NQM) phase is arranged (for arbitrary values of $\nu$ ), in which baryon density is nonzero and chiral symmetry is approximately restored (quark mass is equal approximately to the current quark mass value).

\footnotetext{
${ }^{4}$ In more detail, the influence of $m_{0} \neq 0$ on the duality between CSB and CPC phenomena is investigated in [93] and it is shown there that approximate duality $\mathcal{D}_{H}$ of the phase diagram is observed in the framework of the $\mathrm{NJL}_{4}$ model, and it is a quite good approximation.
} 
This is the sketch of the phase diagram in the physical situation of nonzero current quark mass $m_{0}$. In the chiral limit (zero current quark mass) it is even simpler for in this case the homogeneous PC phase is realized at any nonzero values of isospin chemical potential provided that the value of the quark number chemical potential $\mu$ is not too large.

Assuming that only inhomogeneous CSB phases are possible in the system, the QCDphase diagram was obtained within NJL models, e.g., in [52, 94, 95] (similar finding has been obtained in [51], where this situation was considered in the chiral limit in the quark-meson model). It was found that at rather small values of $\nu(\nu<60 \mathrm{MeV})$ and for $\mu \gtrsim 300 \mathrm{MeV}$ there can exist a region of inhomogeneous CSB (ICSB) phase, namely, CDW ansatz has been considered in these papers, and it was shown that though solitonic modulations are energetically favored against CDW ansatz, it turned out that these changes of ansatz have only mild influence on the phase structure in our model. These calculations were done for simplicity in the chiral limit but probably it is a good approximation. The case of non-zero current quark mass in the case of zero isospin asymmetry was considered in [52] and qualitative picture stays the same. The only changes are that the critical point in the $(\mu, T)$-phase diagram shifts towards smaller temperatures and larger quark chemical potentials with increase of current quark mass and the region of inhomogeneous phase shrinks because its lower border shifts to the larger values of baryon chemical potential. On the other hand, the possibility of the existence of a spatially inhomogeneous charged pion condensation (ICPC) phase of quark matter has been investigated in the framework of $\mathrm{NJL}_{4}$ model, e.g., in ref. [44], where the situation of inhomogeneous CPC and homogeneous CSB phases has been considered. It was shown there that ICPC phase, in which pion condensate exists in a single-plane-wave form, can be realized at rather high value of $\nu$, $\nu \gtrsim 400 \mathrm{MeV}$.

Let us now try to connect these three situations to get full $(\nu, \mu)$ phase diagram. In [95], where ICSB and homogeneous CPC phases have been considered, the CDW was not found in the regions, where CPC phase was considered to be in homogeneous case, and CPC and ICPC phases have been found in [44] (At larger values of $\nu$ the region occupied by ICSB phase decreases but the phase continues to be present at the phase diagram up to the values of $\nu=60 \mathrm{MeV}$ and probably even higher values, there are no plots at larger values in $[94,95]$. It is not clear how far ICSB phase goes to larger values of $\nu$ but it seems it goes over values of $\mu=0.3 \mathrm{GeV}$ almost to the point $\nu=m_{\pi} / 2$ ). And vise versa in [44] there has not been found ICPC phase at rather small values of $\nu$, where ICSB phase is realized in [95]. And if one assumes that there is no mixed phase with ICSB and ICPC condensates, then one can attach these figures and sketch the whole $(\nu, \mu)$ phase diagram in inhomogeneous case. If there is a mixed phase with ICSB and ICPC condensates, then phase diagram can become even more complicated and some regions of normal quark matter phase, homogeneous and/or inhomogeneous phases can be exchanged to the mixed phase (inhomogeneous) and the inhomogeneous phases can become only larger. Putting together the results of the study of the QCD phase diagram, performed in the above mentioned papers [44, 51, 52, 94, 95], etc. both in spatially homogeneous and inhomogeneous approaches to order parameters (condensates), as well as in different areas of chemical potential values, and our above arguments, one can imagine schematically 
the following $(\nu, \mu)$-phase portrait of dense quark matter with isotopic asymmetry that is depicted in figure 1. Note that it corresponds to quark matter, in which chiral asymmetry is absent $\left(\mu_{5}=0, \nu_{5}=0\right)$ and quarks are massive, $m_{0} \neq 0$. Now, let us try to get some information about the phase diagram of dense quark matter when it has chiral asymmetry (in the most general case it means that nonzero values of $\mu_{5}$ and $\nu_{5}$ should be taken into account in the model). Moreover, we suppose that its phase structure is investigated in the framework of a spatially inhomogeneous approach to condensates. If $\mu_{5} \neq 0$, then there is only one way, namely the numerical analysis of the TDP (2.15) or (3.12). This task is rather complicated and has not been solved yet. However, if $\mu_{5}=0$ but $\nu_{5} \neq 0,{ }^{5}$ then, in order to understand how the phase portrait of the model looks, it is not necessary to carry out a numerical study of the TDP (2.15). In this case it is sufficient to perform a dual $\mathcal{D}_{I}$ (3.8) mapping of the phase diagram of figure 1 in order to obtain an approximate $\left(\nu_{5}, \mu\right)$-phase portrait at $\nu=0$ and $\mu_{5}=0$.

Recall that in the chiral limit and in the leading order of the large- $N_{c}$ expansion there is an exact dual symmetry between CSB and CPC phenomena predicted by the $\mathrm{NJL}_{4}$ model (2.1) (see in refs. [15, 70]). As a result, there is an (exact) dual correspondence between some phase diagrams, the consequence of which is the possibility to obtain some phase diagrams of the model without cumbersome numerical calculations, simply acting by the dual transformations $\mathcal{D}_{H}$ (3.3) or $\mathcal{D}_{I}$ (3.8) (see below) on the previously obtained other phase portraits. However, if $m_{0} \neq 0$, then the duality is only approximate symmetry of the TDP, it is observed in the $\mathrm{NJL}_{4}$ model when some of the chemical potentials are intermediate, i.e., greater than $m_{\pi}$ (see in ref. [93]). And the dual correspondence between phase portraits is absent when chemical potentials are small, i.e. $\lesssim m_{\pi}$.

Hence, to get the approximate $\left(\nu_{5}, \mu\right)$-phase diagram of the model (3.9) with $m_{0} \neq 0$ at $\nu=0$ and $\mu_{5}=0$, one just need to take the $(\nu, \mu)$-phase diagram at $\nu_{5}=0$ and $\mu_{5}=0$ and make the following transformations of it: (i) exchange axis $\nu$ to the axis $\nu_{5}$, (ii) outside the region $\omega=\left\{(\nu, \mu): \nu \lesssim m_{\pi}, \mu \lesssim 300 \mathrm{MeV}\right\}$ perform the following renaming of the phases ICSB $\leftrightarrow$ ICPC, CSB $\leftrightarrow$ CPC, and NQM phase stays intact here, and (iii) the phase that lies in the region $\omega$ of the $(\nu, \mu)$-phase diagram should also be present in the region $\tilde{\omega}=\left\{\left(\nu_{5}, \mu\right): \nu_{5} \lesssim m_{\pi}, \mu \lesssim 300 \mathrm{MeV}\right\}$ of the $\left(\nu_{5}, \mu\right)$-phase diagram. The obtained phase diagram is shown in figure 2 and it is called dually $\mathcal{D}_{I}$ conjugated to a phase diagram of figure 1. Since we suppose that current quark mass $m_{0}$ is nonzero (in this case the dual symmetry between CSB and CPC phenomena is only approximate one [93]), the dual $\mathcal{D}_{I}$ mapping of the diagram of figure 1, i.e. the $\left(\nu_{5}, \mu\right)$-phase diagram of figure 2 , presents only an approximate schematic phase portrait of the model (as far as inhomogeneous phases are concerned it is exact). But, nevertheless, it is enough to make several conclusions and predictions about the properties of dense medium with chiral asymmetry.

The obtained phase diagram of figure 2 is quite rich and contains an inhomogeneous CSB phase as well as inhomogeneous CPC phase. It is clear that (inhomogeneous) charged pion condensation phenomenon can be created in the system at a rather small value of $\nu_{5}$

\footnotetext{
${ }^{5}$ As it was discussed in ref. [93] (see also appendix A of the present paper), finite spatial areas with nonzero chiral isospin densities, i.e. with $\nu_{5} \neq 0$, might exist inside compact (neutron) stars due to the chiral separation effect.
} 


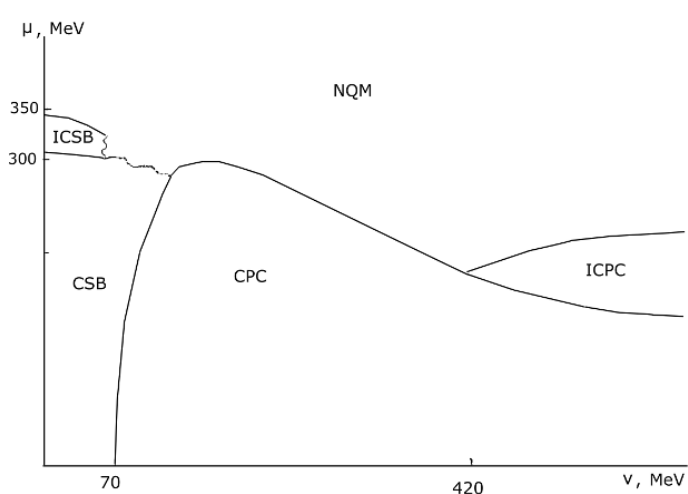

Figure 1. Combined schematic $(\nu, \mu)$-phase diagram at $\mu_{5}=0$ and $\nu_{5}=0$. Here CPC denotes the homogeneous charged pion condensation phase, ICPC denotes the inhomogeneous charged pion condensation phase, CSB and ICSB denotes homogeneous and inhomogeneous phase with broken chiral symmetry, NQM is the normal quark matter phase, where charged pion condensate is zero and quarks have small masses.

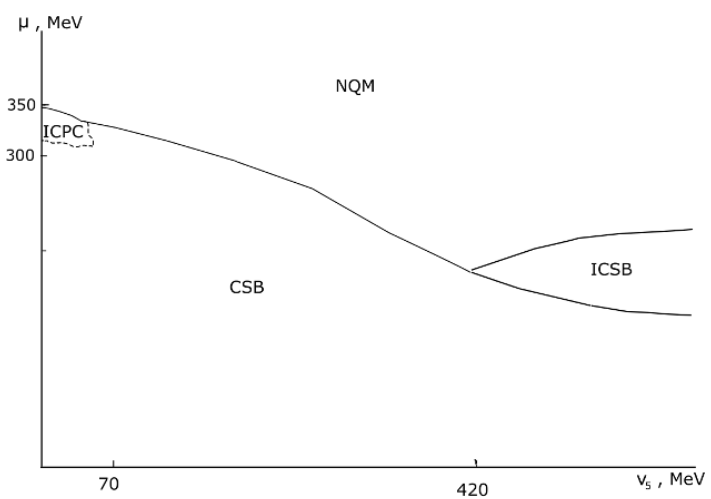

Figure 2. $\left(\nu_{5}, \mu\right)$-phase diagram at $\nu=0$ and $\mu_{5}=0$. This plot is duality conjugated one to a phase diagram of figure 1 . All the notations are the same as in figure 1 . The parts that cannot be drawn with certainty from the duality only are shown with curvy lines. In principle, there could be CPC phase below ICPC one but it is known from other studies that there is no homogeneous CPC phase in this region.

even at zero value of the chemical potential $\nu$. Finally, we see that ICPC phase in figure 2 is located in the region corresponding to rather high values of $\mu$ and, most likely, with nonzero baryon densities. Hence, the chiral isotopic asymmetry in the form of $\nu_{5} \neq 0$ promotes the creation of the CPC phenomenon in dense quark matter. Earlier, this effect was established in the framework of a spatially homogeneous approach to condensates $[15,70,93]$ both in the chiral limit and at $m_{0} \neq 0$, and in the present paper we generalize this conclusion to inhomogeneous case, in addition. Let us add a fly in the ointment. One cannot say about the presence of the ICPC phase with certainty because the duality for homogeneous condensates is an approximate one and it does not work very well in the region of small values of $\nu_{5}$ and $\nu$. And the considerations of [95] have not included the CPC phase, only the chiral symmetry breaking one was considered. It was possible due to the fact that in the physical situation (at the physical point, non-zero current quark mass) the CPC phase is realized only at the values of $\mu_{I}=2 \nu$ larger than the value of the pion mass and in that paper there have been considered only smaller values of $\nu$. So CPC was not expected in this region and there was no need to compare two minima corresponding to the inhomogeneous CSB phase and to the homogeneous CPC phase in the chiral limit. But in the duality conjugated case the homogeneous CSB phase (analog of homogeneous CPC phase in the previous case) can be realized at small values of $\nu_{5}$ and in this case to obtain the full picture one needs to compare these two phases. But let us stress that at least, we know for sure that there is a local minimum point corresponding to ICPC phase (ICPC phase is boosted by chiral imbalance) and there is another one corresponding to CSB phase. To determine which one is the global minimum point, one needs to employ numerical calculations and it 
cannot be studied in terms of duality only. If one assumes that ICPC phase is the global minimum point, then one can see that the chiral isospin chemical potential $\nu_{5}$ generates the charged pion condensation phenomenon even better in the inhomogeneous case. And if not, one can say that inhomogeneous charged pion condensation phase is favoured over the homogeneous one, but still, chiral symmetry breaking phase is a real ground state of the system.

Let us also note that, in principle, there could be homogeneous CPC phase at the phase diagram of figure 2 below the ICPC phase, this fact cannot be figured out just from duality. But from the calculations in the homogeneous case of [93], it is known that homogeneous CSB phase is a more favourable (its minimum lies lower) than homogeneous CPC phase in this region. So, one can conclude that there is no homogeneous CPC phase in this region of the phase diagram, and the only possibility to have other phases in this region (not CSB phase) is inhomogeneous CPC phase. One can also see that chiral isospin chemical potential $\nu_{5}$ can lead to the ICSB phase at values of chemical potential around $0.2 \mathrm{GeV}$ (see figure 2). At first glance, one can think that the quark chemical potential is rather small here, but due to rather large values of chiral isospin chemical potential, at least a part of this phase can possess non-zero baryon density. One can see that at non-zero values of $\nu_{5}$ there seems to be very rich phase diagram featuring as ICSB at rather large values of $\nu_{5}$, as probably ICPC phase at small and moderate values of $\nu_{5}$.

This example shows that duality between CSB and CPC phenomena is not just an interesting mathematical artifact, but a powerful tool in scrutinizing the QCD phase diagram. One knows nothing about the phase structure of inhomogeneous condensates in QCD with chiral isospin chemical potential, does not know even whether it is favoured anywhere in the phase diagram at all, and can get the full phase diagram only due to the use of duality.

\section{Summary and conclusions}

In this paper dense quark matter with isospin and chiral imbalance and dualities (symmetries) of its phase diagram are considered in the framework of $(3+1)$-dimensional NJL model (2.1) in the case of spatially inhomogeneous approach to chiral and charged pion condensates.

Earlier, the phase structure of this model has been studied in details in refs. [15, 70] in the context of spatially homogeneous approach to condensates and in the chiral limit, $m_{0}=0$. In particular, it was shown there that in the leading large- $N_{c}$ order the TDP of the system is invariant under three different dual transformations, $\mathcal{D}_{H}, \mathcal{D}_{H \Delta}$ and $\mathcal{D}_{H M}$ (see in the present section 3.1). One of them, $\mathcal{D}_{H}$, is realized on the phase portrait of the model as a duality correspondence between CSB and CPC phases. It means that if at some fixed values of $\mu, \mu_{5}, \mu_{I}=P$ and $\mu_{I 5}=Q$, e.g., the CSB (or the CPC) phase is realized in the model, then at the dually conjugated values of the chemical potentials, i.e. at the same values of $\mu$ and $\mu_{5}$, but at the permuted values of other chemical potentials, $\mu_{I}=Q$ and $\mu_{I 5}=P$, the CPC (or the CSB) phase must be arranged. So, it is enough to know the phase structure of the model at $\mu_{I}<\mu_{I 5}$, in order to establish the phase structure at $\mu_{I}>\mu_{I 5}$. Knowing condensates and other dynamical and thermodynamical quantities of 
the system, e.g., in the CSB phase, one can then obtain the corresponding quantities in the dually conjugated CPC phase of the model, by simply performing there the duality transformation, $\mu_{I} \leftrightarrow \mu_{I 5}$. Two other symmetries of the TDP, $\mathcal{D}_{H M}$ and $\mathcal{D}_{H \Delta}$, can also impose some restrictions on the shape of the CSB and CPC phases, respectively (see in [15]).

Note that similar dualities have been also considered in the framework of universality principle (large- $N_{c}$ orbifold equivalence) of phase diagrams in QCD and QCD-like theories in the limit of large $N_{c}[86,87]$. In particular, it was noted there that in the chiral limit QCD at $\mu_{I 5} \neq 0$ might be equivalent to QCD at $\mu_{I} \neq 0$, etc (see remarks in section 4 of [86]). Since the Lagrangian (2.1) itself does not have a symmetry that would automatically lead to the dual symmetries of its phase portrait, an interesting question arise whether the duality of the phase portrait obtained in the large- $N_{c}$ limit is a deep property of the theory described by Lagrangian (2.1) or it is just an accidental feature. ${ }^{6}$ In order to get some hints in this direction, the discussed duality between CSB and CPC phenomena has been established both within the homogeneous and inhomogeneous approaches to condensates, but only in the framework of the $\mathrm{NJL}_{2}$ model $[68,69]$ (but in this case the dualities in inhomogeneous and homogeneous approaches are quite similar in terms of proving them, and duality in inhomogeneous case is much easier to show). To confirm these results and to ensure that the duality and related phenomena are intrinsic also to $(3+1)$-dimensional variant of the model (2.1), in the present paper we study the possibility of dual symmetries of its thermodynamic potential using, in contrast to ref. [15], a more extended approach based on the spatially inhomogeneous condensates.

In this paper we have obtained in the leading $1 / N_{c}$ order an exact expression (2.15) of the thermodynamic potential of the model (2.1), when for chiral and charged pion condensates the CDW and single-plane-wave LOFF ansatzes are used, respectively (see eq. (2.10)). A detailed analysis of the phase structure of the model was not carried out in this case. However, we were able to establish that the TDP (2.15) of the system possesses the dual symmetry $\mathcal{D}_{I}(3.8)$, which necessarily leads to a duality between CSB and CPC phenomena. Hence, in the chiral limit both in the homogeneous and more extended spatially inhomogeneous approaches to the ground state of the $\mathrm{NJL}_{4}$ system (2.1), we observe the duality between CSB and CPC phenomena. So, in our opinion, this type of duality is not an artifact of the method of investigation, but the true property of a chirally and isotopically asymmetric dense medium described by the $\mathrm{NJL}_{4}$ Lagrangian (2.1).

Moreover, it is known that when nonzero current quark masses is included in the consideration (at the physical point) the duality is not exact, though it is a good approximation [93]. When one considers the duality in the inhomogeneous case (between inhomogeneous phases) then the duality is exact even at the physical point (see in section IIIC).

It is necessary to bear in mind that in the model (2.1) an arbitrary dual invariance of its TDP, calculated in the approach with homogeneous condensates, is not automatically

\footnotetext{
${ }^{6}$ As a counterexample, we can bring the duality between CSB and superconductivity in some $(1+1)$ and $(2+1)$-dimensional theories [46, 65-67]. But there the original Lagrangians are invariant with respect to the so-called dual symmetry, which includes both the transformation of chemical potentials and coupling constants as well as the Pauli-Gursey transformation of spinor fields (that transforms the chiral interaction channel into a superconducting one, and vice versa). As a result, a duality between these phenomena appears on the phase portrait.
} 
transferred to the case of inhomogeneous condensates. Indeed, the duality between CSB and CPC are realized in the model (2.1) in both approaches, however other dual symmetries, $\mathcal{D}_{H M}$ and $\mathcal{D}_{H \Delta}$, of the TDP $(2.15)$ at $k=0$ and $k^{\prime}=0$ are not observed in the case with inhomogeneous condensates, i.e. at $k \neq 0$ and $k^{\prime} \neq 0$.

In this paper we have not studied the phase portrait in the framework of $(3+1)$ dimensional massless NJL model itself, but we have shown that even if the phase diagram contains phases with nonzero inhomogeneous condensates, it possesses the property of duality (dual symmetry). We demonstrate this fact in terms of TDP in the leading order of the large- $N_{c}$ approximation.

In the literature, the $(\nu, \mu)$-QCD phase diagram has been studied very intensively and it is understood well in homogeneous case. The various aspects of the $(\nu, \mu)$-phase diagram with possible inhomogeneous condensates were investigated in $[44,94,95]$, etc. It has been shown that it is possible to use these shreds and combine them into one unified picture and draw full $(\nu, \mu)$-phase diagram in inhomogeneous case (see in figure 1). When this interesting thing has been completed, from this assembled phase diagram the phase diagram in a completely different situation has been obtained, namely $\left(\nu_{5}, \mu\right)$-diagram of chirally asymmetric QCD matter (see in figure 2). It has been shown that at nonzero values of $\nu_{5}$ there is a very rich phase diagram featuring both the ICSB phase at rather high values of $\nu_{5}$ and the ICPC phase at small and moderate values of $\nu_{5}$. The phase diagram of figure 2 was obtained only by using the duality between CSB and CPC phenomena. Moreover, as it was established in ref. [93], the existence of the duality between CSB and CPC phenomena is supported by lattice QCD results. These instances show that the duality is an inherent property of dense quark matter. So, it is not just entertaining mathematical gaud and interesting but useless mathematical property. In our opinion, it is a potent instrument with very high predictivity power.

It has also been hinted that in inhomogeneous case CPC phase is generated in dense quark matter even by infinitesimally small values of chiral isospin chemical potential $\nu_{5}$. Qualitatively, the same behaviour has been predicted in the framework of $(1+1)$ dimensional NJL model, this concurrence once more consolidates the confidence that $\mathrm{NJL}_{2}$ model can be used as a legit laboratory for the qualitative simulation of specific properties of QCD.

\section{A Generation of nonzero chiral isospin density $n_{I 5}$ in dense quark matter}

Let us suppose, for simplicity, that dense quark matter consists of two massless $u$ and $d$ quarks, whose chemical potentials, $\mu_{u}=\mu+\nu$ and $\mu_{d}=\mu-\nu$ (see the notations adopted just before the eq. (2.4)), are positive. Moreover, we suppose also that quarks do not interact, and there is an external magnetic field $\vec{B}=(0,0, B)$ directed along $z$ axis. In this case in the equilibrium state of quark matter there is a nonzero and nondissipative axial current

$$
\vec{j}_{5 f} \equiv\left\langle\bar{q} f \vec{\gamma} \gamma^{5} q_{f}\right\rangle=\frac{Q_{f} \mu_{f} \vec{B}}{2 \pi^{2}}
$$

for each quark flavor $f=u, d$. In eq. (A.1) $Q_{f}$ is an electric charge of the quark flavor $f$, i.e. $Q_{u}=2 / 3, Q_{d}=-1 / 3$. In this case it is not difficult to conclude from 
eq. (A.1) that axial currents of $u$ and $d$ quarks are opposite in their directions. Since $\vec{j}_{5 f}=\left\langle\bar{q}_{f R} \vec{\gamma} q_{f R}\right\rangle-\left\langle\bar{q}_{f L} \vec{\gamma} q_{f L}\right\rangle$, where

$$
q_{f R}=\frac{1+\gamma^{5}}{2} q_{f}, \quad q_{f L}=\frac{1-\gamma^{5}}{2} q_{f}
$$

we see from eq. (A.1) that left- and right-handed quarks of each flavor $f=u, d$ moves in opposite directions of the $z$ axis. As a result, a spatial separation of quark chiralities for each flavor $f$ occurs. It is the so-called chiral separation effect [7]. In other words, one can say that in the upper half of the three-dimensional space, i.e. at $z>0$, the density, e.g., $n_{u R} \equiv\left\langle\bar{q}_{u R} \gamma^{0} q_{u R}\right\rangle$ of the right-handed $u$ quarks is greater than the density $n_{u L} \equiv\left\langle\bar{q}_{u L} \gamma^{0} q_{u L}\right\rangle$ of the left-handed $u$ quarks. Hence, in this case we have at $z>0$ the positive values of the chiral density $n_{u 5} \equiv n_{u R}-n_{u L}$ for $u$ quarks. (It is evident that at $z<0$ the chiral density of $u$ quarks is negative.)

On the contrary, since the axial current $\vec{j}_{5 d}$ of $d$ quarks differs in its direction from the axial current $\vec{j}_{5 u}$ of $u$ quarks, one can see that in this case at $z>0$ (at $z<0$ ) the chiral density $n_{d 5}$ of $d$ quarks is negative (positive). Consequently, we have at $z>0$ the positive values of the quantity $n_{I 5} \equiv n_{u 5}-n_{d 5}$, which is the ground state expectation value of the chiral isospin density. Whereas at $z<0$ the chiral isospin density is negative.

In summary, we can say that in dense quark medium under the influence of a strong magnetic field (as an example we can mention neutral stars), regions with a nonzero chiral isospin density $n_{I 5}$ might appear. Therefore physical processes inside these regions can be described, e.g., in the framework of the Lagrangians of the form (2.1), containing chiral isospin chemical potential $\mu_{I 5}$.

Open Access. This article is distributed under the terms of the Creative Commons Attribution License (CC-BY 4.0), which permits any use, distribution and reproduction in any medium, provided the original author(s) and source are credited.

\section{References}

[1] Y. Nambu and G. Jona-Lasinio, Dynamical Model of Elementary Particles Based on an Analogy with Superconductivity. 1., Phys. Rev. 122 (1961) 345 [InSPIRE].

[2] Y. Nambu and G. Jona-Lasinio, Dynamical model of elementary particles based on an analogy with superconductivity. II, Phys. Rev. 124 (1961) 246 [INSPIRE].

[3] S.P. Klevansky, The Nambu-Jona-Lasinio model of quantum chromodynamics, Rev. Mod. Phys. 64 (1992) 649 [INSPIRE].

[4] T. Hatsuda and T. Kunihiro, QCD phenomenology based on a chiral effective Lagrangian, Phys. Rept. 247 (1994) 221 [hep-ph/9401310] [INSPIRE].

[5] M. Buballa, NJL model analysis of quark matter at large density, Phys. Rept. 407 (2005) 205 [hep-ph/0402234] [INSPIRE].

[6] K. Fukushima, D.E. Kharzeev and H.J. Warringa, The Chiral Magnetic Effect, Phys. Rev. D 78 (2008) 074033 [arXiv:0808.3382] [INSPIRE].

[7] M.A. Metlitski and A.R. Zhitnitsky, Anomalous axion interactions and topological currents in dense matter, Phys. Rev. D 72 (2005) 045011 [hep-ph/0505072] [INSPIRE]. 
[8] K. Fukushima, Extreme matter in electromagnetic fields and rotation, arXiv:1812.08886 [INSPIRE].

[9] M. Ruggieri, G.X. Peng and M. Chernodub, Chiral medium produced by parallel electric and magnetic fields, EPJ Web Conf. 129 (2016) 00037 [arXiv: 1609.04537] [INSPIRE].

[10] M. Ruggieri, Z.Y. Lu and G.X. Peng, Influence of chiral chemical potential, parallel electric and magnetic fields on the critical temperature of QCD, Phys. Rev. D 94 (2016) 116003 [arXiv: 1608.08310] [INSPIRE].

[11] M. Ruggieri, G.X. Peng and M. Chernodub, Chiral Relaxation Time at the Crossover of Quantum Chromodynamics, Phys. Rev. D 94 (2016) 054011 [arXiv:1606.03287] [INSPIRE].

[12] M. Ruggieri and G.X. Peng, Quark matter in a parallel electric and magnetic field background: Chiral phase transition and equilibration of chiral density, Phys. Rev. D 93 (2016) 094021 [arXiv: 1602.08994] [INSPIRE].

[13] V. Skokov, A.Y. Illarionov and V. Toneev, Estimate of the magnetic field strength in heavy-ion collisions, Int. J. Mod. Phys. A 24 (2009) 5925 [arXiv:0907.1396] [INSPIRE].

[14] A. Bzdak and V. Skokov, Event-by-event fluctuations of magnetic and electric fields in heavy ion collisions, Phys. Lett. B 710 (2012) 171 [arXiv:1111.1949] [INSPIRE].

[15] T.G. Khunjua, K.G. Klimenko and R.N. Zhokhov, Dualities in dense quark matter with isospin, chiral and chiral isospin imbalance in the framework of the large- $N_{c}$ limit of the $N L_{4}$ model, Phys. Rev. D 98 (2018) 054030 [arXiv:1804.01014] [INSPIRE].

[16] D.T. Son and M.A. Stephanov, QCD at finite isospin density, Phys. Rev. Lett. 86 (2001) 592 [hep-ph/0005225] [INSPIRE].

[17] A.W. Overhauser, Structure of Nuclear Matter, Phys. Rev. Lett. 4 (1960) 415.

[18] G.E. Brown and W. Weise, Pion condensates, Phys. Rept. 27 (1976) 1.

[19] A.B. Migdal, Pion Fields in Nuclear Matter, Rev. Mod. Phys. 50 (1978) 107 [InSPIRE].

[20] A.B. Migdal, Stability of vacuum and limiting fields, Zh. Eksp. Teor. Fiz. 61 (1971) 2209 [INSPIRE].

[21] A.B. Migdal, Pi condensation in nuclear matter, Phys. Rev. Lett. 31 (1973) 257 [InSPIRE].

[22] F. Dautry and E.M. Nyman, Pion condensation and the $\sigma$-model in liquid neutron matter, Nucl. Phys. A 319 (1979) 323 [INSPIRE].

[23] G. Gruner, The dynamics of spin-density waves, Rev. Mod. Phys. 66 (1994) 1 [InSPIRE].

[24] A.I. Larkin and Y.N. Ovchinnikov, Nonuniform state of superconductors, Zh. Eksp. Teor. Fiz. 47 (1964) 1136 [INSPIRE].

[25] P. Fulde and R.A. Ferrell, Superconductivity in a Strong Spin-Exchange Field, Phys. Rev. 135 (1964) A550 [INSPIRE].

[26] M.G. Alford, J.A. Bowers and K. Rajagopal, Crystalline color superconductivity, Phys. Rev. D 63 (2001) 074016 [hep-ph/0008208] [INSPIRE].

[27] D. Nickel and M. Buballa, Solitonic ground states in (color-) superconductivity, Phys. Rev. D 79 (2009) 054009 [arXiv:0811.2400] [INSPIRE].

[28] R. Anglani et al., Crystalline color superconductors, Rev. Mod. Phys. 86 (2014) 509 [arXiv: 1302.4264] [INSPIRE].

[29] R. Casalbuoni and G. Nardulli, Inhomogeneous superconductivity in condensed matter and QCD, Rev. Mod. Phys. 76 (2004) 263 [hep-ph/0305069] [INSPIRE]. 
[30] J.A. Bowers and K. Rajagopal, The Crystallography of color superconductivity, Phys. Rev. D 66 (2002) 065002 [hep-ph/0204079] [INSPIRE].

[31] M. Mannarelli, Overview of Crystalline Color Superconductors, in Proceedings, Compact Stars in the QCD Phase Diagram IV (CSQCD IV), Prerow, Germany, September 26-30, 2014 (2015) [arXiv: 1505.04888] [INSPIRE].

[32] D.V. Deryagin, D.Y. Grigoriev and V.A. Rubakov, Standing wave ground state in high density, zero temperature QCD at large $N_{c}$, Int. J. Mod. Phys. A 7 (1992) 659 [inSPIRE].

[33] M. Sadzikowski and W. Broniowski, Nonuniform chiral phase in effective chiral quark models, Phys. Lett. B 488 (2000) 63 [hep-ph/0003282] [INSPIRE].

[34] T. Tatsumi and E. Nakano, Dual chiral density wave in quark matter, hep-ph/0408294 [INSPIRE].

[35] S. Carignano and M. Buballa, Inhomogeneous islands and continents in the Nambu-Jona-Lasinio model, Acta Phys. Polon. Supp. 5 (2012) 641 [arXiv:1111.4400] [INSPIRE].

[36] O. Schnetz, M. Thies and K. Urlichs, Phase diagram of the Gross-Neveu model: Exact results and condensed matter precursors, Annals Phys. 314 (2004) 425 [hep-th/0402014] [INSPIRE].

[37] G. Basar, G.V. Dunne and M. Thies, Inhomogeneous Condensates in the Thermodynamics of the Chiral NJL $L_{2}$ model, Phys. Rev. D 79 (2009) 105012 [arXiv:0903.1868] [INSPIRE].

[38] N.V. Gubina, V.C. Zhukovsky, K.G. Klimenko and S.G. Kurbanov, Emergence of a nonuniform pion condensate in the $(1+1)$-dimensional Nambu-Jona-Lasinio model, Phys. Atom. Nucl. 76 (2013) 1377 [inSPIRE].

[39] V.C. Zhukovsky, K.G. Klimenko and T.G. Khunjua, The effect of chiral density waves on the superconducting phase in the two-dimensional Gross-Neveu model, Moscow Univ. Phys. Bull. 68 (2013) 105 [INSPIRE].

[40] D. Ebert, T.G. Khunjua, K.G. Klimenko and V.C. Zhukovsky, Suppression of superconductivity by inhomogeneous chiral condensation in the NJL model, Int. J. Mod. Phys. A 29 (2014) 1450025 [arXiv:1306.4485] [INSPIRE].

[41] J. Braun, S. Finkbeiner, F. Karbstein and D. Roscher, Search for inhomogeneous phases in fermionic models, Phys. Rev. D 91 (2015) 116006 [arXiv:1410.8181] [InSPIRE].

[42] M. Buballa and S. Carignano, Inhomogeneous chiral condensates, Prog. Part. Nucl. Phys. 81 (2015) 39 [arXiv: 1406.1367] [INSPIRE].

[43] A. Heinz, QCD under extreme conditions: inhomogeneous condensation, Dissertation zur Erlangung des Doktorgrades der Naturwissenschaften, Frankfurt am Main, September (2014).

[44] C.-f. Mu, L.-y. He and Y.-x. Liu, Evaluating the phase diagram at finite isospin and baryon chemical potentials in the Nambu-Jona-Lasinio model, Phys. Rev. D 82 (2010) 056006 [INSPIRE].

[45] N.V. Gubina, K.G. Klimenko, S.G. Kurbanov and V.C. Zhukovsky, Inhomogeneous charged pion condensation phenomenon in the $N J L_{2}$ model with quark number and isospin chemical potentials, Phys. Rev. D 86 (2012) 085011 [arXiv:1206.2519] [InSPIRE].

[46] D. Ebert, T.G. Khunjua, K.G. Klimenko and V.C. Zhukovsky, Competition and duality correspondence between inhomogeneous fermion-antifermion and fermion-fermion condensations in the NJL $L_{2}$ model, Phys. Rev. D 90 (2014) 045021 [arXiv:1405.3789] [INSPIRE]. 
[47] E. Nakano and T. Tatsumi, Chiral symmetry and density wave in quark matter, Phys. Rev. D 71 (2005) 114006 [hep-ph/0411350] [INSPIRE].

[48] V. Schon and M. Thies, Emergence of Skyrme crystal in Gross-Neveu and 't Hooft models at finite density, Phys. Rev. D 62 (2000) 096002 [hep-th/0003195] [INSPIRE].

[49] D. Ebert, N.V. Gubina, K.G. Klimenko, S.G. Kurbanov and V.C. Zhukovsky, Chiral density waves in the $N L_{2}$ model with quark number and isospin chemical potentials, Phys. Rev. D 84 (2011) 025004 [arXiv: 1102.4079] [INSPIRE].

[50] P. Adhikari and J.O. Andersen, Chiral density wave versus pion condensation in the $1+1$ dimensional NJL model, Phys. Rev. D 95 (2017) 054020 [arXiv: 1610.01647] [INSPIRE].

[51] J.O. Andersen and P. Kneschke, Chiral density wave versus pion condensation at finite density and zero temperature, Phys. Rev. D 97 (2018) 076005 [arXiv:1802.01832] [INSPIRE].

[52] D. Nickel, Inhomogeneous phases in the Nambu-Jona-Lasino and quark-meson model, Phys. Rev. D 80 (2009) 074025 [arXiv: 0906.5295] [INSPIRE].

[53] I.E. Frolov, V.C. Zhukovsky and K.G. Klimenko, Chiral density waves in quark matter within the Nambu-Jona-Lasinio model in an external magnetic field, Phys. Rev. D 82 (2010) 076002 [arXiv: 1007.2984] [INSPIRE].

[54] V.C. Zhukovsky, K.G. Klimenko and I.E. Frolov, Density waves in quark matter within the Nambu-Jona-Lasinio model in an external magnetic field, Moscow Univ. Phys. Bull. 65 (2010) 539 [INSPIRE].

[55] E.J. Ferrer and V. de la Incera, Novel Topological Effects in Dense QCD in a Magnetic Field, Nucl. Phys. B 931 (2018) 192 [arXiv:1512.03972] [inSPIRE].

[56] W. Broniowski, Chiral waves in quark matter, Acta Phys. Polon. Supp. 5 (2012) 631 [arXiv: 1110.4063] [INSPIRE].

[57] J. Moreira, B. Hiller, W. Broniowski, A.A. Osipov and A.H. Blin, Nonuniform phases in a three-flavor Nambu-Jona-Lasinio model, Phys. Rev. D 89 (2014) 036009 [arXiv:1312.4942] [INSPIRE].

[58] T. Tatsumi and T. Muto, Quark beta decay in the inhomogeneous chiral phase and cooling of compact stars, Phys. Rev. D 89 (2014) 103005 [arXiv: 1403.1927] [InSPIRE].

[59] K. Nishiyama, S. Karasawa and T. Tatsumi, Hybrid chiral condensate in the external magnetic field, Phys. Rev. D 92 (2015) 036008 [arXiv:1505.01928] [InSPIRE].

[60] A. Heinz, F. Giacosa, M. Wagner and D.H. Rischke, Inhomogeneous condensation in effective models for QCD using the finite-mode approach, Phys. Rev. D 93 (2016) 014007 [arXiv: 1508.06057] [INSPIRE].

[61] T. Kojo, Y. Hidaka, L. McLerran and R.D. Pisarski, Quarkyonic Chiral Spirals, Nucl. Phys. A 843 (2010) 37 [arXiv:0912.3800] [InSPIRE].

[62] T. Kojo, Y. Hidaka, K. Fukushima, L.D. McLerran and R.D. Pisarski, Interweaving Chiral Spirals, Nucl. Phys. A 875 (2012) 94 [arXiv:1107.2124] [InSPIRE].

[63] A. Heinz, F. Giacosa and D.H. Rischke, Chiral density wave in nuclear matter, Nucl. Phys. A 933 (2015) 34 [arXiv: 1312.3244] [INSPIRE].

[64] Y. Takeda, H. Abuki and M. Harada, Novel dual chiral density wave in nuclear matter based on a parity doublet structure, Phys. Rev. D 97 (2018) 094032 [arXiv: 1803.06779] [INSPIRE].

[65] M. Thies, Duality between quark quark and quark anti-quark pairing in $1+1$ dimensional large $N$ models, Phys. Rev. D 68 (2003) 047703 [hep-th/0303026] [INSPIRE]. 
[66] G. Cao, L. He and P. Zhuang, Collective modes and Kosterlitz-Thouless transition in a magnetic field in the planar Nambu-Jona-Lasino model, Phys. Rev. D 90 (2014) 056005 [arXiv: 1408.5364] [INSPIRE].

[67] D. Ebert, T.G. Khunjua, K.G. Klimenko and V.C. Zhukovsky, Competition and duality correspondence between chiral and superconducting channels in $(2+1)$-dimensional four-fermion models with fermion number and chiral chemical potentials, Phys. Rev. D 93 (2016) 105022 [arXiv: 1603.00357] [INSPIRE].

[68] D. Ebert, T.G. Khunjua and K.G. Klimenko, Duality between chiral symmetry breaking and charged pion condensation at large $N_{c}$ : Consideration of an $N J L_{2}$ model with baryon, isospin and chiral isospin chemical potentials, Phys. Rev. D 94 (2016) 116016 [arXiv: 1608.07688] [INSPIRE].

[69] T.G. Khunjua, K.G. Klimenko, R.N. Zhokhov and V.C. Zhukovsky, Inhomogeneous charged pion condensation in chiral asymmetric dense quark matter in the framework of $\mathrm{NJ}_{2}$ model, Phys. Rev. D 95 (2017) 105010 [arXiv:1704.01477] [INSPIRE].

[70] T.G. Khunjua, K.G. Klimenko and R.N. Zhokhov, Dense baryon matter with isospin and chiral imbalance in the framework of $\mathrm{NJL}_{4}$ model at large $N_{c}$ : duality between chiral symmetry breaking and charged pion condensation, Phys. Rev. D 97 (2018) 054036 [arXiv: 1710.09706] [INSPIRE].

[71] C. Ratti and W. Weise, Thermodynamics of two-colour QCD and the Nambu Jona-Lasinio model, Phys. Rev. D 70 (2004) 054013 [hep-ph/0406159] [INSPIRE].

[72] A.A. Andrianov, D. Espriu and X. Planells, An effective QCD Lagrangian in the presence of an axial chemical potential, Eur. Phys. J. C 73 (2013) 2294 [arXiv:1210.7712] [InSPIRE].

[73] R. Gatto and M. Ruggieri, Hot Quark Matter with an Axial Chemical Potential, Phys. Rev. D 85 (2012) 054013 [arXiv:1110.4904] [INSPIRE].

[74] L. Yu, H. Liu and M. Huang, Spontaneous generation of local CP-violation and inverse magnetic catalysis, Phys. Rev. D 90 (2014) 074009 [arXiv:1404.6969] [INSPIRE].

[75] L. Yu, H. Liu and M. Huang, Effect of the chiral chemical potential on the chiral phase transition in the NJL model with different regularization schemes, Phys. Rev. D 94 (2016) 014026 [arXiv: 1511.03073] [INSPIRE].

[76] M. Ruggieri and G.X. Peng, Critical Temperature of Chiral Symmetry Restoration for Quark Matter with a Chiral Chemical Potential, J. Phys. G 43 (2016) 125101 [arXiv:1602.05250] [INSPIRE].

[77] G. Cao and P. Zhuang, Effects of chiral imbalance and magnetic field on pion superfluidity and color superconductivity, Phys. Rev. D 92 (2015) 105030 [arXiv:1505.05307] [InSPIRE].

[78] V.V. Braguta, E.M. Ilgenfritz, A. Yu. Kotov, B. Petersson and S.A. Skinderev, Study of QCD Phase Diagram with Non-Zero Chiral Chemical Potential, Phys. Rev. D 93 (2016) 034509 [arXiv: 1512.05873] [INSPIRE].

[79] N.Y. Astrakhantsev, V.V. Braguta, A.Y. Kotov and A.A. Nikolaev, Lattice study of QCD at finite chiral density: topology and confinement, arXiv:1902.09325 [INSPIRE].

[80] V.V. Braguta, E.M. Ilgenfritz, A.Y. Kotov, A.V. Molochkov and A.A. Nikolaev, Study of the phase diagram of dense two-color QCD within lattice simulation, Phys. Rev. D 94 (2016) 114510 [arXiv: 1605.04090] [INSPIRE].

[81] E. Shuster and D.T. Son, On finite density QCD at large $N_{c}$, Nucl. Phys. B 573 (2000) 434 [hep-ph/9905448] [INSPIRE]. 
[82] E.V. Shuryak, Scales and phases of nonperturbative QCD, in QCD: Perturbative or nonperturbative? Proceedings, 17th Autumn School, Lisbon, Portugal, September 29-October 4, 1999, pp. 210-248 (1999) [hep-ph/9911244] [INSPIRE].

[83] T. Schäfer and F. Wilczek, Superconductivity from perturbative one gluon exchange in high density quark matter, Phys. Rev. D 60 (1999) 114033 [hep-ph/9906512] [INSPIRE].

[84] S. Weinberg, The Quantum Theory of Field II, Cambridge University Press, Cambridge, England (1996).

[85] K. Fujikawa, Path Integral for Gauge Theories with Fermions, Phys. Rev. D 21 (1980) 2848 [Erratum ibid. D 22 (1980) 1499] [INSPIRE].

[86] M. Hanada and N. Yamamoto, Universality of phase diagrams in QCD and QCD-like theories, PoS (LATTICE2011) 221 (2011) [arXiv:1111.3391] [INSPIRE].

[87] M. Hanada and N. Yamamoto, Universality of Phases in QCD and QCD-like Theories, JHEP 02 (2012) 138 [arXiv:1103.5480] [INSPIRE].

[88] D.N. Voskresensky, On the possibility of the condensation of the charged rho meson field in dense isospin asymmetric baryon matter, Phys. Lett. B 392 (1997) 262 [INSPIRE].

[89] J.T. Lenaghan, F. Sannino and K. Splittorff, The Superfluid and conformal phase transitions of two color QCD, Phys. Rev. D 65 (2002) 054002 [hep-ph/0107099] [INSPIRE].

[90] F. Sannino, General structure of relativistic vector condensation, Phys. Rev. D 67 (2003) 054006 [hep-ph/0211367] [INSPIRE].

[91] T. Brauner and X.-G. Huang, Vector meson condensation in a pion superfluid, Phys. Rev. D 94 (2016) 094003 [arXiv: 1610.00426] [INSPIRE].

[92] O. Aharony, K. Peeters, J. Sonnenschein and M. Zamaklar, Rho meson condensation at finite isospin chemical potential in a holographic model for QCD, JHEP 02 (2008) 071 [arXiv: 0709.3948] [INSPIRE].

[93] T.G. Khunjua, K.G. Klimenko and R.N. Zhokhov, Chiral imbalanced hot and dense quark matter: NJL analysis at the physical point and comparison with lattice QCD, Eur. Phys. J. C 79 (2019) 151 [arXiv: 1812.00772] [INSPIRE].

[94] D. Nowakowski, M. Buballa, S. Carignano and J. Wambach, Inhomogeneous chiral symmetry breaking phases in isospin-asymmetric matter, in Proceedings, Compact Stars in the QCD Phase Diagram IV (CSQCD IV), Prerow, Germany, September 26-30, 2014 (2015) [arXiv: 1506. 04260] [INSPIRE].

[95] D. Nowakowski, Inhomogeneous chiral symmetry breaking in isospin-asymmetric strong-interaction matter, Ph.D. Thesis, Technische Univ. Darmstadt (2017). 